\title{
T lymphocyte-derived exosomes and their applications in can- cer therapy
}

\author{
Victor Calvo ${ }^{1}$ and Manuel Izquierdo ${ }^{2, *}$
}

1 Departamento de Bioquímica, Instituto de Investigaciones Biomédicas Alberto Sols CSIC-UAM, Facultad de Medicina, Universidad Autónoma de Madrid, Madrid, Spain; vcalvo@iib.uam.es

2 Instituto de Investigaciones Biomédicas Alberto Sols CSIC-UAM, Madrid, Spain.

* Correspondence: mizquierdo@iib.uam.es; Tel.: +34 914973117

\begin{abstract}
Extracellular vesicles (EV) are a very heterogeneous group of cell-derived vesicles released by almost all kind of living cells. EV are involved in intercellular communication, both locally and systemically, since they induce signals and transfer their contents (proteins, lipids, RNAs) to other cells, which subsequently trigger a wide variety of biological responses in the target cells. However, cell surface receptor-induced EV release is limited to cells from the immune system, including T lymphocytes. $\mathrm{T}$ cell receptor activation of $\mathrm{T}$ lymphocytes induces secretion of $\mathrm{EV}$ containing $\mathrm{T}$ cell receptor for antigen and several bioactive molecules, including proapoptotic proteins. These EV are thus specific for antigen-bearing cells, which make them ideal candidates for a cell-free, EV-dependent cancer therapy. In this review we discuss the generation of EV by $\mathrm{T}$ lymphocytes and some potential therapeutic approaches of these EV.
\end{abstract}

Keywords: exosomes; T lymphocytes; immune synapse; secretory granules; multivesicular bodies; cytotoxic activity; cell death; CAR T lymphocytes

\section{Introduction. Extracellular vesicles types}

Extracellular vesicles (EV) are a very heterogeneous group of cell-derived, lipid bilayer enclosed vesicles released by almost all kind of living cells, and this process is highly conserved throughout evolution [1]. There are various types of EV that have distinct structural, biochemical properties and composition depending of their intracellular origin that in turn affect their function [2]. EV are highly heterogeneous, which in great part is responsible for hindering the characterization and description of their properties and functions [1], and are often classified in terms of their generation mechanism. The first type includes the EV released by dying apoptotic cells, which are called apoptotic bodies. Apoptotic bodies have a wide range of size and exhibit different composition and features from EV derived from living cells, will not be discussed in this review. The second type of EV are directly formed by outward budding of the plasma membrane and are called microvesicles, ectosomes, ectovescicles or, generically, shedding vesicles [3] (Fig. 1). The third type, exosomes, are secreted as a consequence of the fusion of multivesicular bodies (MVB) with the plasma membrane [4] [5] and have diameters ranging 30-150 nm (depending of the estimation technique [6]), smaller than shedding vesicles (100 $\mathrm{nm}$ to $>1 \mu \mathrm{m}$ ) (Fig. 1). MVB are subcellular compartments containing intraluminal vesicles (ILV) [7] [8] that are part of the endosomal system, which also includes early endosomes, late endosomes and lysosomes [9] [10]. MVB are formed by inward budding from the limiting membrane of endosomes and subsequent pinching off of budding vesicles into the luminal space of endosomes (Fig. 1). ILV present in MVB are called exosomes when they are released into the extracellular medium [11]. 


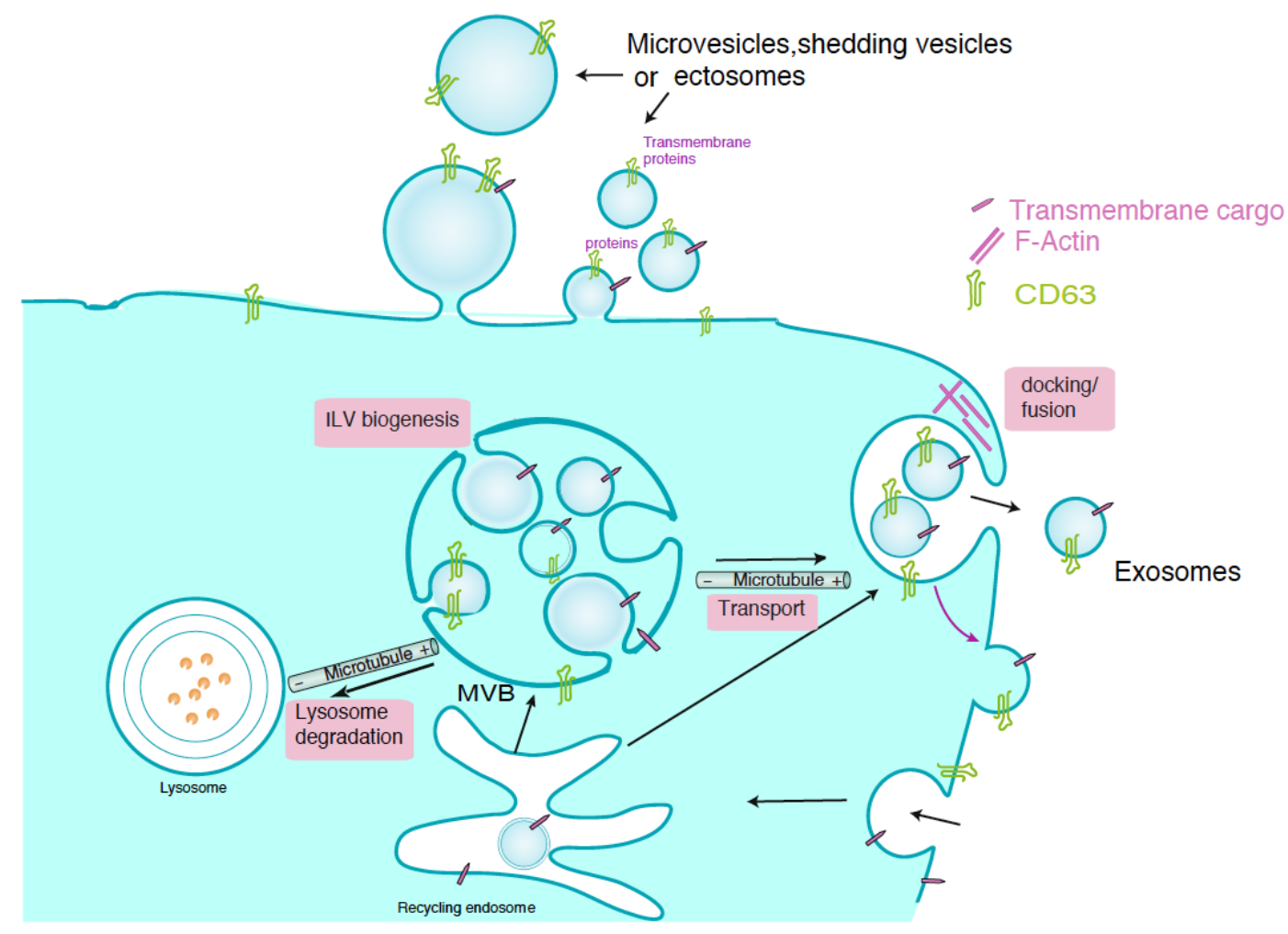

Figure 1. Extracellular vesicles. EV of different intracellular origins can be secreted by eukaryotic cells and the figure represents the different types of membrane vesicles released, either by direct budding from the plasma membrane or by generation of ILV inside MVB, that subsequently fuse with the plasma membrane releasing exosomes. Apoptotic bodies released by dying cells have been excluded. For clarity's sake, only the constitutive secretion of EV and exosomes is represented, although in certain immune cells such as T and B lymphocytes the traffic of MVB and the secretion of exosomes can be induced via T cell receptor (TCR) and B cell receptor (BCR) stimulation [12]. Traffic of MVB comprises three general phases: ILV biogenesis during the maturation of MVB, transport of MVB to the plasma membrane and docking and fusion of MVB to the plasma membrane, whereas EV secretion involves a single step. Transport and fusion of MVB to the lysosomes may lead to MVB degradation. For more details, please refer to [6] [13] [1].

The term exosomes (initially defined to name shedding vesicles with diameters ranging from 40 to $1000 \mathrm{~nm}$ [14]) was later adapted to define nanovesicles of endosomal origin that are released by fusion of MVB with the plasma membrane, as a means to extrude specific obsolete components during red cell maturation [4], and since then this proposal has been widely accepted by the scientific community although not fully standardized yet. However, growing evidences support that EV in general, and exosomes in particular, have much wider biological functions than removal of certain unwanted proteins, and all these EV are involved in intercellular communication, both locally and systemically, since they may transfer their contents (proteins, lipids, RNAs) between cells, and also may trigger new cues in recipient or target cells [10] [15] [1] [12]. Thus, EV have been shown to affect the physiology of neighbouring target cells in diverse manners, from inducing intracellular signaling upon cell surface receptors triggering to conferring new properties to the target cells after acquisition of new receptors, enzymes or even genetic material contained into the EV [16] [2].

\section{Exosomes and extracellular vesicles: normalization attempts and isolation protocols.}

Exosomes are just one type of EV. The International Society of Extracellular Vesicles (ISEV, the major scientific society on EV research, https://www.isev.org/) recommends "extracellular vesicles" as the "generic term for particles naturally released from the cell that are delimited by a lipid bilayer and cannot replicate, i.e. do not contain a functional 
nucleus" [17] including ectosomes, microvesicles, microparticles (different types of plasma membrane-shedding vesicles), apoptotic bodies and exosomes (endosomal origin). Regarding EV characterization, the "minimal experimental requirements for definition of extracellular vesicles" from ISEV or "MISEV" provide recommendations on experimental methodology and minimal information on reporting EV isolation/purification, EV characterization and studies on EV biological function [18] [19]. In this context, although some molecules have been proposed as specific EV markers [18] [17], they do not allow to distinguish among the different EV types [20] $[17,18]$. In conclusion, when an isolated EV preparation is described, the more general term EV is recommended unless the MVB origin of the vesicles it contains has been unambiguously established [13]. We will keep ISEV endorsement of the inclusive term EV along this review, unless the classification of shedding vesicle or exosomes is evident using ISEV criteria.

The isolated material obtained from several EV sources (biological fluids, but also cell culture supernatants) generally contains variable proportions of the diverse EV and the composition of the recovered EV may vary vastly depending of the protocol [18] [13]. Therefore, as the first consideration, given the heterogeneity within the preparations, it is problematic to assign a biological effect to any EV contained in the preparations. Accordingly, "MISEV" recommendations include biochemical (i.e. protein markers), biophysical (i.e. single vesicle characterization by electron microscopy -EM- and nanoparticle tracking analysis -NTA-) and functional tools (i.e. EV depletion to remove biological activity) that may allow researchers to ascribe any potential function to $\mathrm{EV}$.

As the second issue, although numerous analyses have highlighted proteins, miRNAs and lipids commonly found in exosomes and EV preparations, even curated by ISEV standards (see EXOCARTA: http://exocarta.org/index.html\# and VESICLEPEDIA: http://www.microvesicles.org/ ) it is clear that these studies do not denote "exosome-specific" markers or "EV-specific" markers, but rather "exosome-enriched" (or "EV-enriched") proteins. For instance, CD63 and CD81, which are reported to be enriched in exosomes produced by a wide variety of cell types studied (EXOCARTA: exosomal markers) and have been considered as canonical exosome markers [13], are also present at the plasma membrane of $\mathrm{T}$ and $\mathrm{B}$ lymphocytes, most probably as a consequence of the diffusion of the CD63 located at the limiting membrane of MVB upon their constitutive fusion with the plasma membrane [21] [22] and hence located in shedding vesicles (and, quite probably, in apoptotic bodies) (Fig. 2). Considering this caveat, the EV field has proposed a list of EV-specific markers [18] [17], but has not achieved to distinguish among EV subtypes [20], such as those produced either via shedding from the plasma membrane or via endosomal compartments [17,18]. In this context, although MISEV 2018 recommendations do not define an universal negative marker, for any given EV source, markers should be chosen to reveal the level of "contaminants" in that specific EV material [17] [23]. This may be applied to different EV subtypes, by establishing specific markers of their subcellular origin that are reliable within the experimental system [17]. Accordingly, some molecules can be used as negative markers for certain EV types and particular cell types. As an example, the evaluation of both positive exosome markers (CD63 and lysosomal-associated membrane protein 1 -LAMP-1-) and negative markers (i.e. CD45 and CD28, which are very abundant in the $\mathrm{T}$ lymphocyte plasma membrane and hence in plasma-membrane derived EV and apoptotic bodies, but absent in exosomes [24]) in EV preparations isolated by differential centrifugation allowed characterization of the isolated EV as canonic exosomes [25]. A similar approach was followed by other researchers in order to characterize as bona fide exosomes the EV produced by chimeric antigen receptor (CAR)

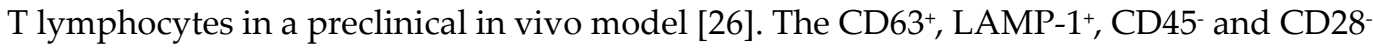
$\mathrm{T}$ lymphocyte-derived exosomes were subsequently confirmed in their endosomal/MVB origin by analyses of CD63+ MVB fusion with the plasma membrane in living T cells [21].

A third consideration relies on the fact that different EV in the diameter range of exosomes share biophysical characteristics such as size, membrane topology and density [13], and thus current isolation methodology and biophysical analyses cannot offer unambiguous characterization unless complementary biochemical and biophysical experiments 
are performed (i.e. density gradient centrifugation, western blot, EM, single particle analyses and phenotyping by NTA, nano flowcytometry, etc.) [27]. Moreover, the overlapping size distributions among different $\mathrm{EV}$ and the absence of clearly-standarized, discriminatory molecular markers, often hinder the assignment of isolated EV to one of these classes in experimental settings [3].

In conclusion, EV samples most probably contain a mixture of EV of exosomal and non-exosomal nature, therefore unless their MVB origin has been unambiguously established, it is recommended to use the more general term EV [13] and we will follow this criterion in this review.

\section{Exosome biogenesis, composition and regulation of exosome secretion.}

Several researchers have demonstrated the endosomal origin of exosomes by using EM [10] [28] or fluorescence living-cell imaging [21]. Solid evidence of their endosomal origin is often difficult to obtain, since MVB fusion with the plasma membrane is a very dynamic and stochastic process [22] that is difficult to document using EM. Despite this caveat, several researchers have obtained definitive data regarding their MVB origin by using EM [10] [28] or fluorescence living-cell imaging [21] [22]. ILV are formed by inward budding of the membrane of late endosomes at least via three distinct molecular mechanisms, which have been partially characterized (Fig. 2). It is out of the scope of this review to extensively explain these mechanisms, please refer to excellent published reviews on this subject [29] [6] [30] [1]. Therefore, we first will discuss MVB and exosome generation mechanisms in general terms, and subsequently we will focus on the mechanisms operating in T lymphocytes.

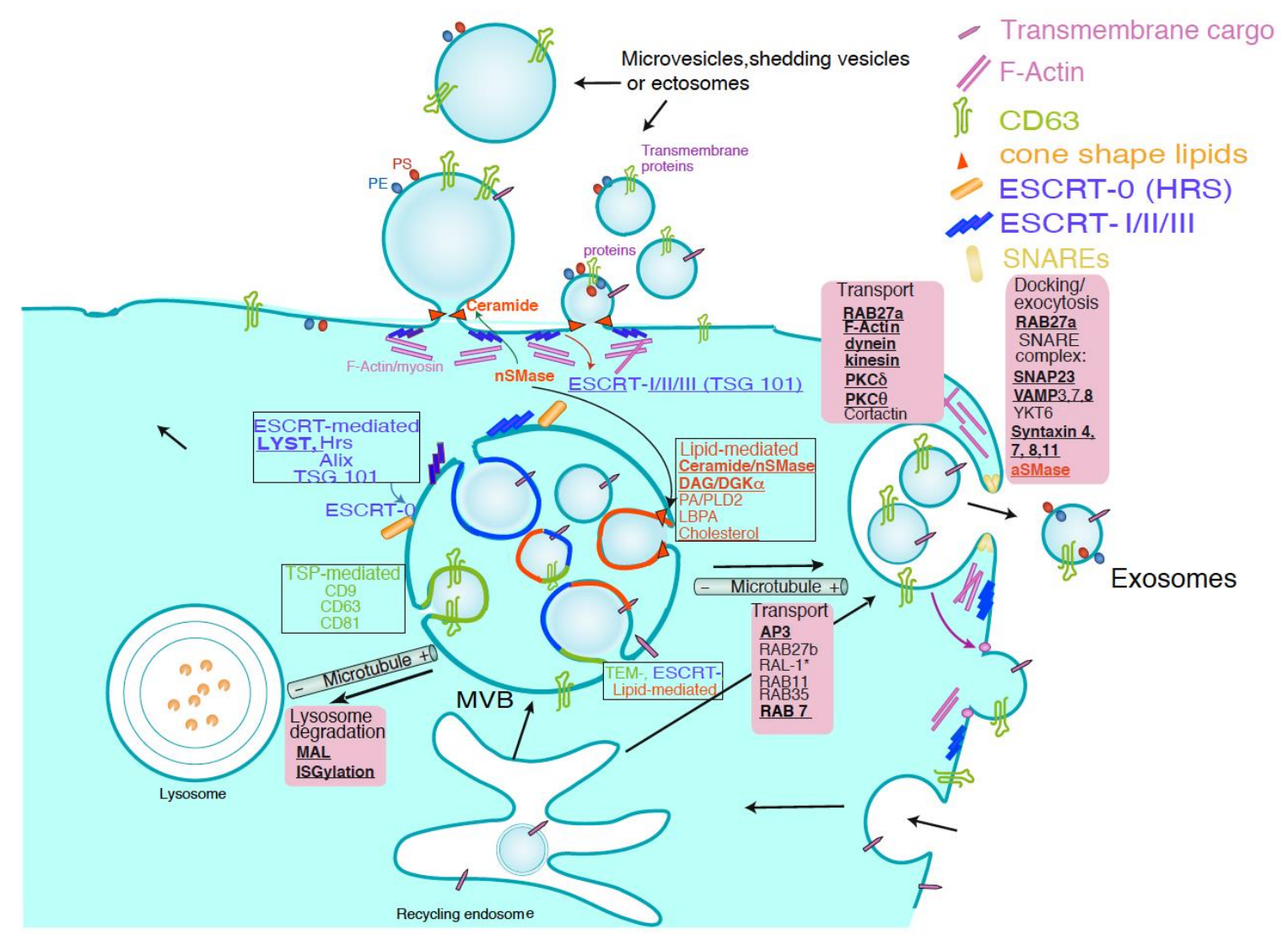

Figure 2. General and T lymphocyte-specific mechanisms of shedding vesicles and exosome biogenesis and MVB traffic. The inward, intraluminal budding of specific membrane nanodomains from the MVB limiting membrane produces ILV. The invagination of ILV and the sorting of specific cargoes can be produced by the action of three mechanisms that are enclosed in black line boxes: the Endosomal Sorting Complex Required for Transport (ESCRT)-0-I-II-III machinery (blue), 
tetraspanins (green) or certain lipids as cholesterol, ceramide, diacylglycerol (DAG) and lysobisphosphatidic acid (LBPA) (red). In addition, multiple machineries (represented as mixed colors) can collaborate in ILV biogenesis. It is unclear whether the three mechanisms simultaneously act on the same MVB or each one acts on different MVB, although all mechanisms are shown operating in the same MVB for clarity's sake. Black line rectangles enclose the general mechanisms involved in exosome biogenesis, whereas the regulators of MVB traffic (including transport to lysosomes for degradation, transport to the plasma membrane, docking and fusion with the membrane) are enclosed in magenta boxes. ESCRT-0 components (hepatocyte growth factor-regulated tyrosine kinase substrate -Hrs-, STAM) are generally not observed in plasma membrane budding leading to shedding vesicles, whereas ESCRT-I-II-III are involved in these processes (reviewed in [13]). However, both ESCRT-0 and ESCRT-I-II-III are involved in ILV formation inside MVB [13] [1]. Depolymerization of the actin cytoskeleton is required for secretion of shedding vesicles and exosomes. In addition, externalization of phosphatidylethanolamine (PE) and phosphatidylserine (PS), that may bind Annexin V, occurs in plasma membrane-derived EV secretion and, to a lower extent, in exosome secretion.

Bold, underlined characters identify those molecular components that regulate MVB secretory traffic in T lymphocytes: lysosomal trafficking regulator (LYST) [31], neutral sphingomyelinase 2 (nSMase2) [32], DAG [33], diacylglycerol kinase $\alpha$ (DGK $\alpha)[21,25,34]$ [35], acidic sphingomyelinase (aSMase) [36], MAL [30,37], ISGylation [38], Adaptor protein 3 (AP3) [39], Rab27a [40], Rab11, Rab7 [41], dynein [42], kinesin-1 [43], cortical F-actin [44] [45], centrosomal area F-actin [46] [47], protein kinase C $\delta$ (PKC $\delta$ ) [48] [45], protein kinase C $\theta$ (PKC $\theta$ ) [49] [50], vesicle-associated membrane protein 8 (VAMP-8) [51], syntaxin 4 (STX4) [52], syntaxin 7 (STX7) [53], syntaxin 8 (STX8) [54], syntaxin 11 (STX11) [55], SNAP23 [52]. Underlined characters identify molecules involved in shedding vesicles generation in T lymphocytes: tumor susceptibility gene 101 (TSG101) and vacuolar protein sorting 4 (VPS4) [56]. LYST has Hrs (an ESCRT-associated protein) as binding partner, which supports that LYST participates in MVB biogenesis [57] [58].

The first mechanism controlling ILV generation involves ESCRT members [7]. However, exosomes can also be formed in a ESCRT-independent manner since MVB containing ILV loaded with CD63 are still formed upon depletion of the components of the four ESCRT complexes (reviewed in [1]). The second mechanism involves the action of certain lipids such as ceramide [59] [60] [30] and DAG [34] [21]. This first ESCRT-independent mechanism of exosome biogenesis requires the generation of ceramide by nSMase2, which hydrolyses sphingomyelin to ceramide [60]. Ceramide, a cone-shaped lipid, may then allow the generation of membrane subdomains, which impose a spontaneous negative curvature on the limiting membrane of late endosomes favoring inward budding of ILV (Fig. 2). Moreover, purified exosomes were enriched in ceramide, and the release of exosomes was reduced after the pharmacologic inhibition of nSMase or nSMase interference [60]. Vice versa, inhibitors of sphingomyelin synthase (i.e. D609) that enhance ceramide levels have been shown to increase exosome secretion [61]. Inverted cone-shaped lipids such as LBPA are also abundant in internal membranes (ILV) of MVB [62]. LBPA controls the formation of ILV both in vitro and in vivo through the recruitment of ESCRT-family member Alix [63]. Thus, interconnection between ESCRT-dependent and ESCRT-independent, lipid-mediated pathways may exist (Fig. 2) [6]. In addition, phospholipase D2 (PLD2), activated by ARF6 small GTPase, produces the hydrolysis of phosphatidylcholine to phosphatidic acid (PA), a cone-shaped lipid as ceramide, which has been shown to be necessary for ILV biogenesis and exosome secretion [64] [65]. The model proposed is that PA formation in the inner leaflet of MVB limiting membrane would induce inward curvature and thus formation of ILV [64], as described for ceramide [60]. In addition, cholesterol confers high fluidity to lipid bilayers and is necessary for the formation of highly curved membrane structures such as caveolae and synaptic vesicles. In this context, caveolin-1, has been recently shown to act as a cholesterol rheostat in MVB, regulating ILV biogenesis and exosomal protein cargo sorting through the control of cholesterol content at the endosomal compartment/MVB and hence regulating exosome secretion [66]. 
The third mechanism for exosome biogenesis involves proteins of the tetraspanin (TSP) family that have been shown to regulate ESCRT-independent endosomal sorting (reviewed in [6] [1] [30]. Tetraspanins such as CD63, CD9 and CD81 form clusters with other tetraspanins and with different transmembrane proteins and certain lipids in membrane domains known as tetraspanin-enriched microdomains. Apparently, tetraspanins facilitate the formation of the membrane microdomains that will undergo inward budding to facilitate ILV formation [1] [30]. For instance, CD81 has a cone-shaped structure with an intramembrane cavity that accommodates cholesterol and that is likely to be shared by other tetraspanins. Clustering of these cone-shaped tetraspanins/cholesterol complexes could then induce the inward budding of the microdomains in which they are enriched [1], which constitutes an example of crosstalk between tetraspanin and lipid-mediated mechanisms involved in ILV biogenesis [6]. In addition, CD63 may also trigger ESCRTindependent and ESCRT-dependent endosomal sorting, which is another example of crosstalk during MVB biogenesis [67] (Fig. 2). MAL is a membrane protein containing four transmembrane segments and its expression is restricted to T lymphocytes and polarized epithelial and myelin-forming cells. MAL appears also to be responsible for ILV biogenesis and exosome secretion in T lymphocytes, since MAL silencing in T lymphocytes reduces constitutive exosome secretion and generates immature, aberrant MVB that do not fuse with the plasma membrane and merge with lysosomes [30,37] (Fig. 2). Another pathway that regulates MVB traffic towards lysosomes for degradation is ISGylation (Fig. 2). Interferon stimulated gene 15 (ISG15) is an ubiquitin-like protein and ISG15-dependent ISGylation of ESCRT family member TSG101 protein induces its aggregation and degradation, being sufficient to impair constitutive exosome secretion in T lymphocytes, although inducible, TCR-controlled exosome secretion was not evaluated in these experiments [38].

Apart from ILV biogenesis, the transport, docking and fusion of mature MVB with the plasma membrane are crucial steps involved in the secretory traffic of exosomes, that are strictly regulated. It is out of the scope of this review to deal in depth with all the pathways, molecular components and mechanisms involved in MVB polarized traffic, so please refer to recent and excellent reviews on this subject for details [6] [1] [13]. MVB may fuse either with lysosomes (Fig. 2) for degradation of their content or with the plasma membrane. In both cases, three steps (transport, docking and fusion) are required, but the effectors involved in targeting MVB to lysosomes or the plasma membrane are distinct. In general, the intracellular transport of MVB involves the association of these organelles with the cytoskeleton (actin filaments and microtubules), associated molecular motors (dynein, kinesin, etc.) and molecular switches (Rab GTPases) [1]. In brief, there are three major group of regulators of MVB transport and fusion with the plasma membrane, that include Rab GTPases, actin/tubulin cytoskeleton and soluble N-ethylmaleimide-sensitive fusion attachment protein receptors (SNAREs) [13] [1]. Among these, Rab GTPase members control different steps of intracellular vesicular traffic, such as vesicle budding, vesicle and organelle mobility through cytoskeleton interaction and vesicle docking to target compartments, leading to membrane fusion, and play a very conserved role during MVB traffic to the plasma membrane in diverse cell types (reviewed in [65] [6] [68]). The strategies directed to unveil the role of Rab GTPases in exosome secretion are mainly based on overexpression of dominant negative mutants or silencing by using small hairpin RNA (shRNA) [65] [6]. Thus, loss of function approaches to interfere with diverse Rab GTPases (Rab 7, Rab 11, Rab 27a, Rab27b and Rab35) in different cell types have resulted in inhibition of MVB transport and exosome secretion, although particular Rabs may act in different cell types [65] [6] (Fig. 2). The microtubule network is required for MVB transport to the plasma membrane for subsequent exosome release [68] [13] (towards microtubule plus ends in the centrosome, at least in the context of the immune synapse-IS), although MVB can also be targeted towards lysosomes via retrograde transport on microtubules (towards microtubule minus ends) thanks to the retrograde molecular motor dynein (Fig. 2) [1]. Regarding actin cytoskeleton, depolymerization of the cortical F-actin at the central IS is necessary to allow MVB docking to the plasma membrane for subsequent exosome 
release [45] and, in T and B lymphocytes, the dismantling of F-actin pool around microtubule-organizing center (MTOC) and MVB is involved in MTOC/MVB polarization [69] [46] [70]. In the last step of exosome secretion, SNAREs (synaptosomal protein 23 SNAP23-, VAMP7, VAMP8, YKT6, syntaxins) are specifically required for calcium-dependent MVB fusion with the plasma membrane (reviewed in [1]). Thus, the molecular components involved in these stages have been partially identified and have been shown to be common, but may also differ, among different cell types [1] [68].

All the mechanisms for MVB maturation also control, during ILV formation from the limiting endosomal membrane, that certain proteins, lipids, and nucleic acids are specifically sorted to ILV, and subsequently constitute exosome cargo (reviewed in [6]) (Fig. 2). Please refer to the ISEV standards-curated, web pages regarding proteins, miRNAs and lipids commonly found and enriched in exosomes and other EV (EXOCARTA: http://exocarta.org/index.html\# and VESICLEPEDIA: http://www.microvesicles.org/). Exosome cargo reflects in part the composition of the donor cell but is also a consequence of a regulated sorting mechanism [1]. Exosomal cargo comprises various proteins including receptors, transcription factors, transmembrane proteins, enzymes, extracellular matrix proteins, but also lipids and nucleic acids (DNA, mRNA, and miRNA), inside and on the surface of exosomes [1] (Fig. 3).

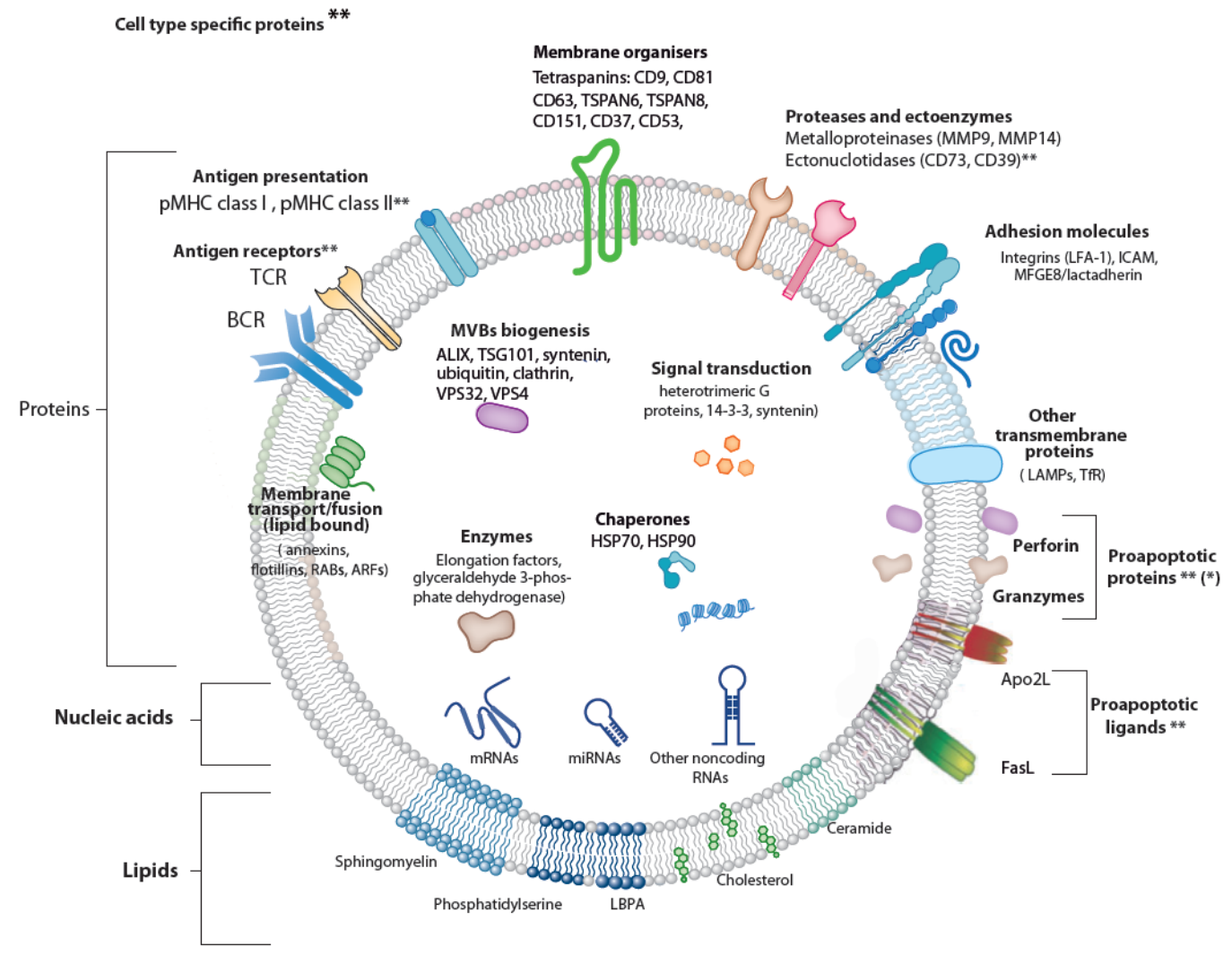

Figure 3. Exosome structure and molecular composition. Exosomes are surrounded by a phospholipid bilayer and contain nucleic acids and proteins (grouped by biological function), lipids, and nucleic acids. Exosomal proteins: annexins, important for transport; tetraspanins and integrins important for cell targeting and binding; Alix and TSG101, involved in exosomal biogenesis from endosomes. Abbreviations: FLOT1, flotillin1; HSP, heat shock protein; MHC, major histocompatibility complex; RabGDI, RabGDP-dissociation inhibitor; RAP1B, Ras-related protein1B; TSG101, tumor susceptibility gene 101. $\left.{ }^{* *}\right)$ labels those proteins which are specifically found in exosomes produced by $\mathrm{T}$ or B lymphocytes, whereas the rest of the indicated proteins are mostly found with high frequency $(>30 \%)$ in exosomes produced by different cell types [2]. $\left(^{*}\right)$ It is not clear whether perforin/granzymes are located or not inside exosomes. For more details regarding exosome composition visit http://www.exocarta.org. 
Analysis of exosome protein composition revealed that some proteins are common among exosomes from different cellular origins, whereas some proteins are specific of cells and tissues that secrete them [71] [1]. Regarding protein exosome composition, some exosomal proteins are present in exosomes from different cell types, whereas other exosomal proteins are cell type-specific [71] [1]. Exosomal proteins common to different cell types include adhesion molecules such as cell adhesion molecules (CAM), integrins, tetraspanins (CD63, CD9, CD81), MHC-I, a range of membrane fusion and transferring proteins (Rab2, Rab5, Rab7, flotillin 1, annexins), heat shock proteins (HSP70, HSP90), cytosolic proteins involved in MVB formation (Alix, Tsg101) and cytosolic enzymes (GAPDH) [72] [1] [73] (see also EXOCARTA http://exocarta.org/ )(Fig. 2). MHC-II is specific of B lymphocytes and antigen-presenting cells (APC)-derived exosomes [74], and transferrin receptors (TfR) are specific of reticulocyte-derived exosomes [1]. BCR and TCR are specific of B or T lymphocyte-derived exosomes, respectively [75] [24]. Fas ligand (FasL) and Apo2 ligand (Apo2L) appear to be more restricted to T lymphocyte-derived exosomes [74] [76] and exosomes from certain tumor cells [77]. Perforin and granzyme A and B have been shown to be associated with exosomes from cytotoxic T lymphocytes (CTL) and natural killer cells (NK) [78] [79] [80]. It is not clear whether perforin/granzymes reside inside ILV or, alternatively, are externally-associated to ILV membrane [78] [79] (Fig. 3). However, it has been shown by exosome surface labelling and flow cytometry [81], that the later situation may occur in chimeric antigen receptor (CAR) T-cell derived exosomes, facilitating perforin-granzyme binding and activity on target cells [26] [81]. If this is confirmed for different CAR T-cell and T-cell derived exosomes, it will be necessary to understand how these cytosolic proteins are sorted or translocated to the luminal side of the ILV. The fact these proapoptotic proteins are not integral membrane proteins would suggest that association to ILV limiting membrane and hence to exosomes could be mediated by unknown membrane adapter protein(s). Alternatively, if perforin/granzyme are located inside exosomes, further research is necessary to understand how these proteins are functionally delivered to target cells.

In addition, the lipid content of exosomes is both cell-specific and common. Lipids not only have a significant role in maintaining exosome shape and curvature but also participate in exosome biogenesis (see above). LBPA interaction with Alix facilitates the inward budding of MVB limiting membrane [63], which constitutes an example of crosstalk between ESCRT and lipid-mediated mechanisms involved in ILV biogenesis (Fig. 2). Sphingomyelin, phosphatidylcholine and LBPA help distinguishing numerous types of vesicles. Different types of microvesicles have a similar content of sphingomyelin and phosphatidylcholine while sphingomyelin concentration is higher in exosomes. LBPA is exclusive to endosomes and exosomes [82]. During membrane rearrangements occurring during EV generation there are changes in the lipid asymmetry of membrane phospholipids (PS exposition from the inner to the outer leaflet of the lipid bilayer) (Fig. 2), leading to Annexin-V binding, that was mainly associated with the microvesicles and, to a lower extent, with exosomes [83]. However, PS exposure also occurs in apoptotic bodies [84], therefore Annexin-V binding cannot be used to distinguish among different EV types.

Adding more complexity to exosome composition, it has been established that the same type of cells releases distinct exosome subpopulations with unique compositions that may elicit differential effects on recipient cells [85]. Such exosome subpopulations possibly originate from different MVB subpopulations and the distinct compositions of these subpopulations probably reflect the presence of multiple sorting machineries (i.e ESCRT-dependent and independent, see above) that act on the MVB compartment [1] (Fig. 2). Further dissection of exosome heterogeneity will advance our understanding of exosomal biology in health and disease. In this context, most commonly used methods allow bulk analysis of vesicles (i.e. western blot with EV markers), but are not suited for accurate quantification and fail to reveal phenotypic heterogeneity in membrane vesicle populations. For such analyses, the development of multiparameter, high-throughput analysis methods at the single vesicle level is needed (i.e. [86]) [87] [88,89]. Regarding lipid exosome composition, sphingomyelin concentration is higher in exosomes than in other 
vesicles, and LBPA is exclusive to endosomes and exosomes [82]. Inverted cone-shaped lipids like LBPA $[62,90]$ in the external leaflet of the MVB lipid bilayer and ceramide, DAG, as well as PA in the internal leaflet [64], may facilitate negative curvature of the lipid bilayer needed for ILV formation and thus exosome formation [91] [60] [7].

Regarding lipids role in shedding vesicle formation, nSMase has been shown to be involved in plasma membrane budding in several cell types [92] [13]. nSMase inhibitors or its interference reduce exosome secretion by blocking the ceramide-dependent inward ILV budding into MVB lumen [92]]. In contrast, nSMase blockade stimulates EV budding at the plasma membrane [92]. Most probably, this is due to the asymmetric lipid distribution in the external and internal membrane leaflets and thus sphingomyelin hydrolysis to ceramide might lead to more or less EV or exosomes depending on the membrane leaflet that is positively or negatively curved by sphingomyelin to ceramide conversion [93] [91] [92].

\section{Extracellular vesicles from T lymphocytes}

IS formation by T lymphocytes upon TCR binding to antigen bound to APC is a very dynamic, plastic and critical event involved in antigen-specific, cellular and humoral immune responses [94] [95]. IS establishment integrates signals and coordinates molecular interactions leading to an appropriate and antigen-specific immune response [96]. There are two major groups of IS built by T lymphocytes leading to very different, but essential immune effector functions $[94,96,97]$. The interaction of helper T lymphocytes (Th), generally CD4+ cells, with MHC-II-bearing APC results in T lymphocyte activation (cytokine secretion, proliferation, etc.). In contrast, naïve CTL, usually CD8+ cells, recognize antigen-associated to MHC-I on APC and then become activated or "primed" to proliferate in the first phase, and kill target cells bearing the antigen in the second, effector phase. In the effector phase, primed CTL also form IS with target cells (cells infected by viruses or tumor cells) resulting in specific killing. Thus, the functional changes produced by the formation of a productive, mature IS include activation (naïve CTL and Th lymphocytes), killing (primed CTL), and functional anergy or apoptosis induction [98] once effector phase is finished. IS formation induces the convergence of T lymphocyte secretory granules towards the MTOC and, almost simultaneously, the polarization of the MTOC and the secretory granules towards the central supramolecular cluster (cSMAC) at the IS $[95,99]$. T lymphocyte secretory granules include cytokine-containing secretion granules in Th lymphocytes, cytolytic granules/secretory lysosomes in CTL, and MVB in Th lymphocytes and CTL. This specialized mechanism appears to specifically provide the immune system with a finely-tuned tactic to increase the efficiency of crucial secretory effector functions of T lymphocytes, while minimizing nonspecific, cytokine-mediated stimulation, target cell killing and activation induced cell death (AICD) of bystander cells [100].

CTL cytolytic granules are secretory lysosomes that have MVB structure and their degranulation cause ILV secretion as nanosize "extracellular vesicles" at the CTL-target cell synaptic cleft, first described by Peters et al. [101]. Although the vesicles secreted by CTL were not referred at that time as canonic exosomes, their formation and mode of exocytosis justifies this classification [10] (see above). Cytolytic granules contain perforin and granzymes that are located into a lumenal, electron dense core characterized by EM and also accumulate in ILV [78]. In addition, it was shown that ILV and their derived exosomes contained, apart from the proapoptotic proteins perforin and granzymes, the exosome marker CD63 and molecules relevant for CTL-target cell interaction such as TCR and CD8 [101] [78], demonstrating that most of the cytotoxic mediators exocytosed into the cleft between CTL and the target cell are membrane-enveloped or exosome-associated. However, release of perforin and granzymes in soluble form coming from the lumenal core cannot be excluded [78] [102]. Perforin, which is inactive in the acidic environment of secretory lysosomes, is activated by neutral $\mathrm{pH}$ and $\mathrm{Ca}^{2+}$ at the synapse polymerizes and forms a transmembrane pore that allows the entry of granzymes into the target cell, and granzymes are involved in triggering caspase-dependent and independent cell death 
[58]. It was early hypothesized that the presence of the TCR complex, CD8, and possibly other relevant molecules on these nanovesicles displaying their extracellular portions facing outwards may ensure unidirectional delivery of lethal factors to target cells, since it was proposed that ILV released into the synaptic cleft bind specifically to the relevant antigen-MHC-I complex on the target cell membrane, and not to the CTL itself or to bystander cells [79]. This model would explain not only why a CTL does not kill itself, but also why bystander cells, which are in close proximity but do not bear the proper antigen, are not killed [79]. Subsequently, it was demonstrated that newly synthesized Fas ligand (FasL) is also stored in the limiting membrane of CTL secretory lysosomes and that polarized degranulation controls FasL delivery to the T cell surface, which was consistent with a role of a FasL-dependent pathway in CTL-mediated cytotoxicity [103].

In addition, it was shown that FasL can also be sorted from MVB limiting membrane to ILV and hence to exosomes upon T lymphocyte activation, since T lymphocyte activation induced the secretion of 100-200 nm "microvesicles" containing proapoptotic FasL and Apo2L [104] (Fig. 3). These microvesicles were subsequently characterized as canonic exosomes, since they derived from FasL ${ }^{+} \mathrm{Apo}_{2} \mathrm{~L}^{+} \mathrm{ILV}$ upon MVB fusion with the plasma membrane [28]. Exosomal FasL and Apo2L, with the same topology as cell surface FasL and Apo2L, can bind to their respective death receptors on the surface of target cells, or effector T lymphocytes themselves, inducing caspase-dependent apoptosis [105] [106] [107] (Fig.4). Proapoptotic exosomes are thus involved in AICD of effector T lymphocytes, which constitutes an important suicide or fraticide mechanism participating in the downregulation of T cell-dependent immune responses [104] [28] [108]. Another major contribution was to demonstrate that inducible exosome secretion occurred in a polarized manner at the IS formed by living Th lymphocytes and APC [21], as occurred in the IS formed by CTL [101] [78] (Fig. 4).

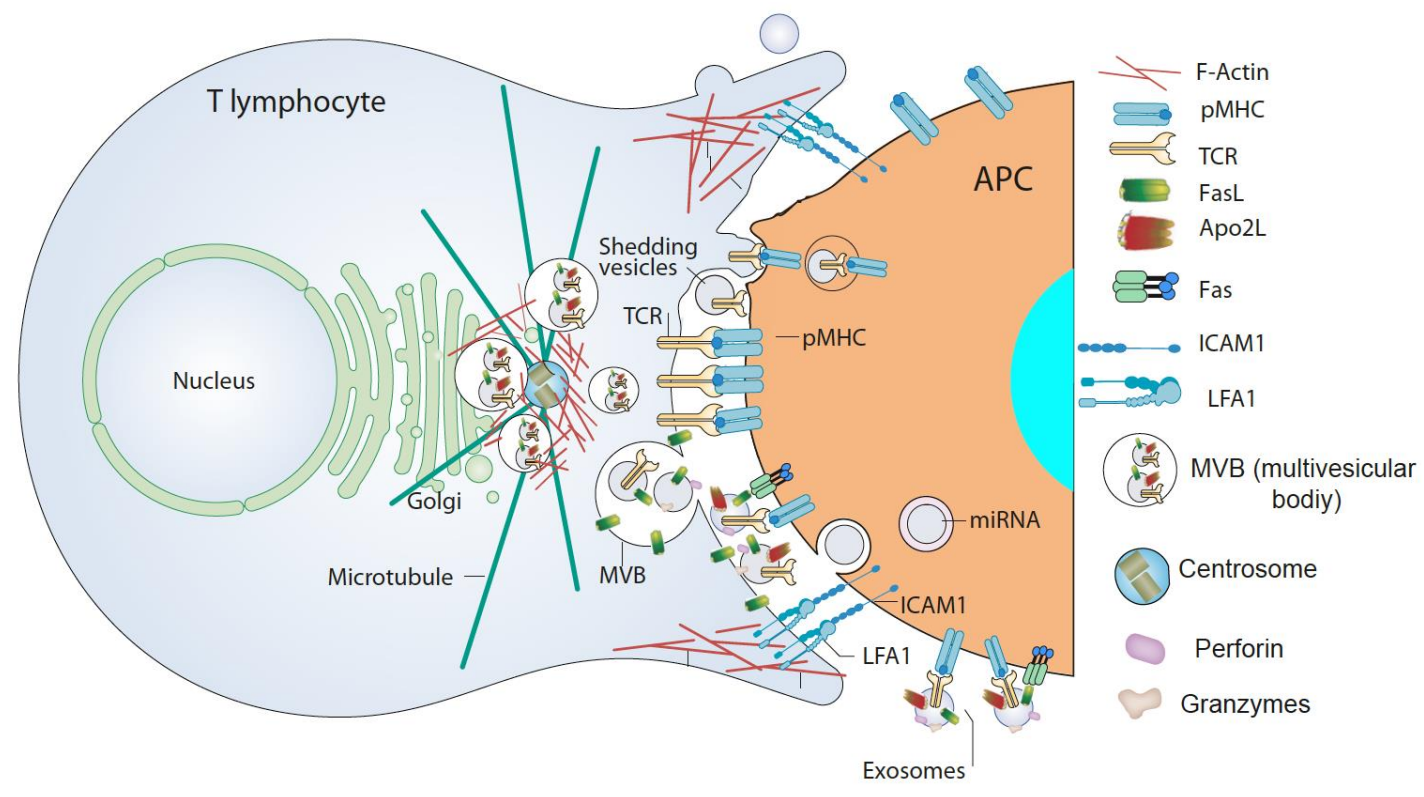

Figure 4. EV in the immune synapse. In a mature IS produced by TCR stimulation via the peptideMHC complex (pMHC) on the APC and the interaction of accessory molecules (such as Intercellular Adhesion Molecule 1 -ICAM1- with Lymphocyte function-associated antigen 1 -LFA-1-) F-actin is reduced at the central region of the IS that corresponds to cSMAC, F-actin accumulates into the distal SMAC (dSMAC) and F-actin surrounding the centrosome depolymerizes. All these processes, most probably acting in a coordinated manner, may facilitate centrosome movement towards the IS and the simultaneous convergence of MVB towards the F-actin depleted area in the CSMAC, facilitating MVB fusion at the CSMAC, and the subsequent secretion of exosomes carrying TCR and proapoptotic molecules in the synaptic cleft towards the APC. In addition, shedding vesicles emerging from the plasma membrane and containing TCR are represented at the synaptic cleft. Both 
exosomes containing miRNA [32] and shedding vesicles [109] [110] are engulfed by APC and provide biological responses in APC. For more details please refer to [12,70] [111] [109].

The hypothesis derived from all these publications that TCR activation of the effector T cell may induce the release of $\mathrm{CD}^{2} 3^{+}$exosomes bearing TCR was formally demonstrated by using TCR agonists to activate Jurkat Th, CTL and CD4+ lymphocytes[24] [25]. Taken together, these reports constitute a major milestone in the exosome field since they demonstrate that TCR stimulation triggers inducible exosome secretion by T lymphocytes (both in CTL and Th cells) [2] [12]. However, it is remarkable that, depending on the stimulation regime (absence or presence of co-stimulation signals) $\mathrm{CD}^{+} \mathrm{T}$ cell activation promotes the differential release of distinct EV subpolulations [112].

Adding more complexity to the EV field, CD63-enriched shedding vesicles or ectosomes directly budding from the Th lymphocyte plasma membrane and accumulating at the IS formed with a B lymphocytes acting as APC have been described [56] (Fig. 4). These synapse-induced shedding vesicles were enriched in TCR and, upon endocytosis by APC, were capable to signal via pMHC-II stimulation [56] [113] (Fig. 4), suggesting these synaptic ectosomes may facilitate the activation of B cells and other APC presenting the cognate pMHC-II. In this report, centrally accumulated TCR are located on the surface of extracellular microvesicles that bud at the IS centre. Members of the ESCRT-I family such as TSG101 sort TCR for inclusion in shedding vesicles, whereas VPS4 mediates microvesicle scission from the T-cell plasma membrane. TSG101 interference reduced EV production, whereas VPS4 function disruption rendered budding vesicles unable to undergo fission from the plasma membrane [56] (Fig. 4). However, neither the existence of T cell-derived shedding vesicles in the CTL IS nor a proapoptotic role of these EV has been demonstrated yet. In addition, although nSMase has been shown to be involved in plasma membrane budding in several cell types [92] [13], its participation in EV release from T lymphocytes has not been reported yet, in contrast with its role in ILV biogenesis [60].

\section{Traffic of cytotoxic granules and MVB in Tymphocytes}

The molecular studies of CTL from patients with inherited defects leading to immunopathological haemophagocytic lymphohistiocytosis (HLH) syndrome and their mouse models that impaired CTL function mediated by defects in secretory lysosomes/cytolytic granules have highlighted the importance of cytotoxicity in the regulation and termination of immune responses. The characterization of these defects has contributed to our understanding of the key molecular steps that are required for the maturation and traffic of cytolytic granules and the secretion of their contents at the IS during target cell killing by CTL [114] [58]. Included among the genetic disorders associated with the occurrence of HLH, mutations affecting lysosomal trafficking regulator (LYST) affect biogenesis of cytolytic granules [31], whereas AP3 mutations affect lytic granule polarization [39]. In addition, mutations in Rab27a affect docking of lytic granules to the synaptic membrane [115] [40], and mutations in STX11 [55], MUNC13-4 [116] and MUNC18-2 [117] inhibit lytic granule degranulation by exocytosis. Perforin is essential for lytic granule-mediated cytotoxicity, as indicated in studies from perforin-deficient mice and humans that carry inherited mutation of the genes encoding perforin [118]. All of these genetic alterations produce defects in CTL and NK cytotoxic function through the cytolytic granule-dependent pathway, resulting in severe HLH [114] [58]. Not surprisingly, several regulators of cytolytic granules polarized traffic and fusion to the plasma membrane in Tymphocytes include some of the conserved molecular components of MVB traffic pathways, such as Rab27a [40] and Rab7 [41] (Fig. 2). In addition, several members of the SNARE family, such as syntaxins 4, 7, 8 and 11 [52] [53] [54] [55], have been described to be involved in lytic granule exocytosis in CTL, the latest stage of MVB traffic (Fig. 2).

Regarding the molecular machineries specifically involved in MVB maturation and exosome biogenesis and secretion in Th lymphocytes, there is little information available in comparison to other cell types, including CTL, probably due to the fact that, besides the constitutive and non-polarized secretion, TCR triggering induces directional exosome 
secretion at the synapse [2] [30] [12], and this fact hinders experimental approaches. In fact, analysis of EV secretion at steady state or upon TCR activation may influence the mechanism of exosome secretion so that specific experimental conditions (steady state versus stimulation or costimulation) may affect the results [13]. Probably, the lipid pathway is the best characterized mechanism involved in exosome biogenesis and secretion in $\mathrm{T}$ lymphocytes. In this regard, silencing of nSMase 2 or inhibition of its enzymatic activity that decreases ceramide production induce a decrease in the release of exosomes [32]. Some other lipid-metabolizing enzymes controlling $\mathrm{T}$ cell activation such as diacylglycerol kinase $\alpha(\mathrm{DGK} \alpha)$ [119], that reduces DAG levels by producing PA, accumulate in MVB limiting membrane and control MVB generation and CD63-enriched exosome release in T lymphocytes [34] [21,25]. DAG, as well as ceramide and, to a lower extent, PA, are cone-shaped lipids that when located in the inner leaflet of MVB limiting membrane can induce inward curvature and thus ILV formation [65]. Thus, pharmacologic inhibition of DGK $\alpha$ enhances MVB maturation and exosome secretion [25] [21]. The finding that Hrs (ESCRT-0 family member) is not required for inducible exosome release by Tymphocytes [32] indicate that, although other ESCRT components have not been tested yet, exosome biogenesis appears to be independent of ESCRT machinery in T lymphocytes, unlike in other cell types [120] [121] [122]. Moreover, CTL from aSMase-deficient (aSMase-KO) mice are defective in exocytosis of cytolytic effector molecules upon IS formation; this defect resulted in attenuated cytotoxic activity of aSMase-KO CTL [36]. Thus, aSMase is required for contraction of secretory granules and expulsion of cytotoxic effectors, including exosomes, in CTL [36] (Fig. 2). This reveals the importance of ceramide biosynthesis, for both exosome biogenesis and the late stages of MVB traffic during inducible exosome secretion at the IS. Taken together, it appears that several components of the lipid pathways involved in ceramide and DAG/PA metabolism may be strong candidates for a pharmacologic or genetic intervention directed to modulate inducible exosome secretion by $\mathrm{T}$ lymphocytes.

Regarding the traffic of MVB and cytolytic granules towards the plasma membrane in T lymphocytes forming IS, MVB follow the network of microtubules oriented by the MTOC, and exosome and lytic granule secretion is thus provided by the inducible oriented traffic of MVB and the MTOC towards the IS [123] [45]. Therefore, it is remarkable that secretory polarized traffic leading to exosome secretion is an inducible and unique feature occurring in both CTL and Th lymphocytes IS upon challenge with antigen [12]. Thus, probably due to the intrinsic attributes of polarity and inducibility, pMHC-stimulated TCR evokes finely-tuned, lipid-regulated specific signalling pathways leading to MVB polarization. In this context, there is a considerable number of studies on the characterization of the signals involved in MVB polarization, docking and fusion to the synaptic membrane, in comparison with the scarce reports on MVB maturation in T lymphocytes (reviewed in [12]). Exosome secretion is provided by the MTOC/secretory granules reorientation towards the IS in CTL and Th lymphocytes, that is initially guided by the DAG gradient centered at the IS, generated by TCR-stimulated phospholipase C (PLC) $[33,95]$. DAG phosphorylation by DGK $\alpha$ is involved in the negative, spatiotemporal control of the DAG gradient [119] and MTOC/secretory granule polarization to the IS in CTL and Th lymphocytes [33]. DGK $\alpha$ inhibition enhances T cell activation by upregulating DAG levels [119]. DAG activates several members of the PKC, such as PKC $\theta$ and PKCठ, and protein kinase D (PKD) families [124]. PKC $\theta$ facilitates MTOC polarization by localizing dynein anchored to Adhesion and Degranulation Promoting Adapter Protein (ADAP) in the F-actin rich areas at the IS, which pulls the MTOC forwards [50] [49] [125]. PKCO is necessary for cytolytic granule polarization and cytotoxicity in mouse CTL [48] and for MVB polarization in Th IS [45]. In a Th-APC IS model, a positive role of TCRtriggered DAG and a negative role of the DAG regulator DGK $\alpha$ [119] in polarized MVB traffic towards the IS has been demonstrated [34]. These findings, together with the negative control that DGK $\alpha$ exerts on MTOC and cytolytic granule polarized traffic in CTL (described above) support that DAG, several DAG-activated PKC isoforms and DGK $\alpha$ 
can be considered as general regulators of secretory polarized traffic in T lymphocytes [49] [35] [12].

Several evidences support an important role of actin cytoskeleton reorganization in exosome secretion triggered upon T lymphocyte IS (reviewed in [126]). Not surprisingly, several actin cytoskeleton regulators such as actin-related proteins 2/3 (ARP2/3), hematopoietic lineage cell-specific protein 1 (HS1), transgelin-2 (TAGLN2), Diaphanous-related formin 1 (Dia1) and Formin-like 1 (FMNL1) have been shown to participate in MTOCguided, polarized secretory traffic at the IS [127] [128,129] [130], although the contribution of all these actin cytoskeleton regulators specifically to exosome secretion has not been addressed yet. Since several recent reviews have dealt with the contribution of actin cytoskeleton reorganization to polarized secretory traffic at the IS [131] [132] [70], we will focus on some relevant issues directly related to exosome secretion. F-actin depolymerization at the IS center controls MTOC and secretory granule polarization to the secretory domain of both CTL [123] [44] and Th lymphocytes IS [133] [134]. Regarding signals involved in actin reorganization, DAG-activated PKC $\delta$ is needed for cortical actin reorganization at the IS, MTOC/MVB polarization to the IS and exosome secretion by Th lymphocytes IS [45]. Thus, cortical F-actin depolymerization at the central IS appears to be necessary to allow MVB docking to the plasma membrane for subsequent exosome release [13] This supports that an altered actin reorganization at the IS may underlie the deficient MVB polarization occurring in PKC $\delta$-interfered Th lymphocytes [45]. More recently, PKC $\delta$-dependent depolymerization of the F-actin pool surrounding the MTOC/MVB (Fig. 4) has been shown to be involved in MTOC/MVB polarization to the Th IS [46] [70] [47]. It is well known that microtubule network is required for the transport of late endosomes, since MTOC co-migrates with diverse secretory granules (cytolytic granules /MVB in CTL, cytokine secretory granules/MVB in Th lymphocytes) [123] [46] [13]. Thus, a crosstalk between actin remodeling and the microtubule network exists during polarized traffic at the IS [126], which would render the microtubule and/or actin cytoskeletons as pharmacologic targets to inhibit or to enhance exosome secretion. For instance, cortical F-actin cytoskeleton depolymerization may indirectly promote exosome secretion. However, due to the involvement of actin and tubulin cytoskeleton in a myriad of crucial cellular functions, targeting these networks is also likely to induce diverse non-specific effects [13].

In summary, pharmacologic or genetic DGK inhibition might be used therapeutically to enhance inducible exosome secretion by T lymphocytes [12]. DGK $\alpha$ inhibition also enhances DAG levels at the early stages of T cell activation [119]. Thus DGK $\alpha$ inhibition, by increasing both $\mathrm{T}$ cell activation and inducible exosome secretion $[21,25,34]$, boosts exosome secretion and might be useful for cell-free, exosome-based therapies (see below).

\section{Chimeric Antigen Receptor (CAR) T cells and CAR T cell-derived EV. Cancer thera- peutic approaches}

The role of EV from immune cells, including $\mathrm{T}$ lymphocytes, in anti-tumor immunity has been recently and exhaustively reviewed [135] [136] [137], thus we will focus on potential therapeutic uses of CAR T cell-derived EV. CAR T lymphocyte-based immunotherapy has been proved to become a promising treatment of patients suffering several refractory cancer diseases [138]. CAR consists of an extracellular domain, which confers antigen-recognition specificity, a transmembrane domain, and an intracellular signaling do-

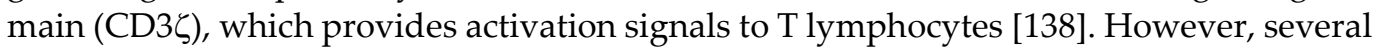
challenges preclude the use of adoptively-transferred CAR T cells, including their low efficacy against solid tumors, immunosuppression by tumor microenvironment, poor $\mathrm{T}$ cell persistence, $\mathrm{T}$ cell dysfunction or exhaustion and cytokine release syndrome (CRS) [138] [139]. It is out of the scope of this review to summarize the preclinical and clinical uses of CAR T cells for cancer therapy (there are more than 250 clinical trials testing CAR $\mathrm{T}$ cells, please refer to recent and excellent reviews on this subject [138] [139]), thus we will focus on trials using CAR T cell-derived EV. 
Considering the early hypothesis that the presence of both TCR and proapoptotic molecules (FasL, Apo2L, perforin, granzymes A and B) on T cell-derived exosomes would endow them with both antigenic specificity and directional cytotoxicity $[78,79,101]$, making them powerful vectors to deliver proapoptotic signals to target cells bearing the cognate antigen, several strategies have been developed to test the use of CAR T cell-derived exosomes for cancer therapy. The fact that TCR activation boosts the production of CTLderived exosomes, and the presence of the TCR/CD3 $\zeta$ complex in these exosomes (see above) [24] would reinforce this approach. TCR/CD3 $\zeta$ complexes endocytosed after recognition of the pMHC-II complexes are targeted to MVB, then to ILV and hence exosomes [24]. A crucial prerequisite for using CAR T cell-derived exosomes to specifically induce tumor cell death is the presence on exosomes of the CAR molecule since its antibodyderived, antigen-binding variable fragment would endow them with tumor cell specificity, as occurs with CAR T cells [138] [140]. Since CAR is an artificial molecule containing the $\mathrm{CD} 3 \zeta$ intracellular signaling and localization domain, it was unknown whether CAR was present in exosomes and shedding vesicles, and whether CAR conferred a specific cytotoxic effect, until recent reports characterized CAR expression and function in exosomes [26] and shedding vesicles [141]. This prerequisite has been validated in several preclinical studies by using exosomes and/or EV derived from CAR T cells [26] [81] [142] (Table 1).

Table 1. CAR T cell and cell-derived EV: preclinical studies.

\begin{tabular}{|c|c|c|c|c|c|}
\hline Target molecule & $\begin{array}{c}\text { EV-producing } \\
\text { cell }\end{array}$ & EV types & $\begin{array}{l}\text { EV pheno- } \\
\text { type }\end{array}$ & $\begin{array}{l}\text { Anti-tumor } \\
\text { mechanism }\end{array}$ & Target cell \\
\hline $\begin{array}{c}\text { EGFR, HER2 } \\
{[26]}\end{array}$ & $\begin{array}{c}\text { Human CAR T } \\
\text { cells } \\
(?)^{1}\end{array}$ & Exosomes & $\begin{array}{c}\mathrm{CAR}^{+}, \mathrm{CD}^{+}, \\
\mathrm{CD}^{+} 3^{+}, \\
\text {perforin } \\
\text { granzyme } \\
\mathrm{B}^{+}, \\
\mathrm{CD}^{+} 5^{-} \\
\mathrm{CD}^{-}\end{array}$ & $\begin{array}{c}\text { Perforin/ } \\
\text { granzyme B² }\end{array}$ & $\begin{array}{c}\text { EGFR }^{+}, \\
\text {HER2 }^{+} \\
{ }^{2} \text { human breast } \\
\text { cancer cells }\end{array}$ \\
\hline $\begin{array}{c}\text { HER2 } \\
{[142]}\end{array}$ & $\begin{array}{c}\text { Human CAR T } \\
\text { cells } \\
\text { CD4+(46\%) } \\
\text { CD8+(49\%) }\end{array}$ & $\begin{array}{l}\text { EV (small EV, } \\
\text { probably exo- } \\
\text { somes } \\
\text { plus larger } \\
\text { EV) })^{3}\end{array}$ & $\begin{array}{l}\mathrm{CAR}^{+}, \mathrm{CD}^{+}, \\
\mathrm{CD}^{+} 3^{+}, \\
\text {granzyme B }{ }^{+}\end{array}$ & $\begin{array}{c}\text { Granzyme } \\
\mathrm{B}^{2}\end{array}$ & $\begin{array}{l}\text { HER2 }^{+} \\
\text {human breast } \\
\text { cancer cells, } \\
\text { ovarian can- } \\
\text { cer cells }\end{array}$ \\
\hline $\begin{array}{l}\text { Mesothelin } \\
\text { [81] }\end{array}$ & $\begin{array}{c}\text { Human CAR T } \\
\text { cells } \\
\text { CD4+(58\%) } \\
\text { CD8+(31\%) }\end{array}$ & $\begin{array}{l}\text { Probably exo- } \\
\text { somes }^{4}\end{array}$ & $\begin{array}{c}\mathrm{CAR}^{+}, \mathrm{CD}^{+}, \\
\mathrm{CD}^{+}{ }^{+}, \text {per- }^{-} \\
\text {forin } \\
\text {, } \\
\text { granzyme } \mathrm{B}^{+}\end{array}$ & $\begin{array}{c}\text { Perforin/ } \\
\text { granzyme B² }\end{array}$ & $\begin{array}{l}\text { Triple nega- } \\
\text { tive } \\
{ }^{2} \text { human breast } \\
\text { cancer cells }\end{array}$ \\
\hline $\begin{array}{l}\text { CD19 } \\
{[143]}\end{array}$ & $\begin{array}{l}\text { Human CAR } \\
\text { HEK293 cells }\end{array}$ & $\begin{array}{l}\text { Probably exo- } \\
\text { somes }^{4}\end{array}$ & $\begin{array}{l}\mathrm{CAR}^{+}, \\
\mathrm{CD}^{+}{ }^{+} \\
\mathrm{CD}^{+} 1^{+}\end{array}$ & $\begin{array}{l}\text { Indirect in- } \\
\text { duction of } \\
\text { proapoptotic } \\
\text { genes in tar- } \\
\text { get cells }\end{array}$ & $\begin{array}{l}\mathrm{CD} 19+^{+} \text {human } \\
\text { B cell leuke- } \\
\text { mia }\end{array}$ \\
\hline $\begin{array}{l}\text { CD19 } \\
{[141]}\end{array}$ & $\begin{array}{l}\text { Human CAR } \\
\text { HEK293 cells }\end{array}$ & $\begin{array}{c}\text { Probably } \\
\text { shedding vesi- } \\
\text { cles }^{4}\end{array}$ & $\begin{array}{c}\mathrm{CAR}^{+}, \text {an- } \\
\text { nexin } \mathrm{V} \\
\text { binding } \\
\end{array}$ & $\begin{array}{l}\text { MYC Gene } \\
\text { disruption } \\
\text { mediated by }\end{array}$ & $\begin{array}{l}\text { CD19+ human } \\
\text { B cell leuke- } \\
\text { mia cell lines }\end{array}$ \\
\hline
\end{tabular}




\begin{tabular}{|c|c|c|c|c|}
\hline & & & $\begin{array}{c}\text { (PS expo- } \\
\text { sure) }\end{array}$ & $\begin{array}{c}\text { CRISPR/Cas } \\
9\end{array}$ \\
\hline $\begin{array}{l}\text { Mesothelin } \\
\text { CD19 [144] }\end{array}$ & $\begin{array}{c}\text { Human and } \\
\text { mouse CAR T } \\
\text { Cells } \\
(?)^{1}\end{array}$ & $\mathrm{EV}^{4}$ & $\begin{array}{c}\text { Unknown } 5 \\
\text { Contain } \\
\text { RN7SL1 }\end{array}$ & $\begin{array}{l}\text { Recruitment } \\
\text { of endoge- } \text { Mouse mela- } \\
\text { nous anti-tu- } \text { noma ex- } \\
\text { mor immun- } \\
\quad \text { pressing hu- } \\
\text { byRN7SL1 }\end{array}$ \\
\hline
\end{tabular}

${ }^{1}$ No data available regarding $\mathrm{CD} 4{ }^{+} / \mathrm{CD}^{+}$subpopulations. ${ }^{2}$ Proposed mechanism, but not formally demonstrated. ${ }^{3}$ Unfractionated $\mathrm{EV}$; more criteria to distinguish exosomes from shedding vesicles are needed. ${ }^{4}$ More criteria to distinguish exosomes from shedding vesicles are needed. ${ }^{5}$ No phenotypic analyses of EV were performed.

CAR T cell-derived exosomes exhibit excellent potential for use as direct attackers in immunotherapy, since ex vivo-produced human exosomes carrying human EGFR and HER2-specific CAR have potent in vivo activity against EGFR ${ }^{+}$and $\mathrm{HER}^{+}$human tumor cells in xenograft models [26]. These CAR T cell-derived exosomes specifically induce apoptosis of tumor cells expressing the antigens recognized by CAR on the cell surface but do not kill tumor cells that do not express these antigens [26]. Comparable results were obtained using HEK-derived exosomes, showing that only when exosome entry into cells is mediated via binding to the CD19 antigen on the surface of CD19+ B-cells, proapoptotic signaling occurs that results in selective cytotoxicity [81] [142]. Certainly, the evoked apoptotic mechanism does not depend on FasL, Apo2L, perforin and granzymes, as for CAR T-cell derived exosomes, since HEK293 cells do not express these molecules. More recently, an interesting paper has demonstrated an in vivo role of RNA component of signal recognition particle 7SL1 (RN7SL1, a non-coding RNA that activates interferonIFN-stimulated genes)-containing EV from CAR T cells in tumor rejection [144]. In this approach, the CAR construct contains downstream of the CAR sequence a U6 promoter that drives the transcription of human RN7SL1 and, optionally, a 5'LTR promoter driving the transcription of a peptide antigen. The authors demonstrate that RN7LS1-containing CAR T cells deliver RN7SL1 in EV in vivo, that orchestrates endogenous immune activation to improve response against the tumor. EV released by CAR T cells upon CAR engagement transfer RN7SL1 to endogenous immune cells (myeloid cells, DC and T cells), but not to the tumor cells, and EV release and RN7LS1 transfer is inhibited by a nSMase inhibitor. RN7LS1 inside target cells activates a RIG-I-dependent, IFN-mediated inflammatory response and induces transcription of IFN-stimulated genes. RN7LS1 delivery in EV to immune cells improves immunostimulatory properties of myeloid and DC cells, that in turn effectively activate the function of endogenous CD8 T cells against the tumor. All these immune cells, acting together, may trigger solid tumor rejection even in case of CAR-recognized antigen loss by the tumor. Although in this research neither EV nature or composition, nor the presence and contribution of CAR and proapoptotic proteins in the EV to tumor rejection have been established, this strategy opens new venues based on improved endogenous immunity against tumor cells conferred by EV, since CAR T cells can now co-deploy antigenic peptides with RN7SL1 released by EV to enhance their efficacy against tumor cells, even when tumors lack adequate neoantigens [144]. Table 1 summarizes the most relevant features of the preclinical trials involving CAR $\mathrm{T}$ cell and HEK293-derived EV. Most of these trials have been performed ex vivo or in vivo using xenograft models in mice.

The advantages of CAR T cell-derived exosomes as cell-free immunotherapy are their independence of CAR T cell life span and replication, their stability, obvious logistic issues, the low risk of side toxicity (i.e., CRS incidence) when compared to CAR T cells, and the fact that exosomes lacking PD-1 (in contrast to PD-1-expressing T cells) are refractory to PD-L1 immunosupression by the tumor [26]. In this context, is remarkable that CAR T 
cell-induced CRS is one of the most adverse events that follows infusion of the CAR T cells and occurs in nearly two thirds of CAR T cell recipients and usually occurs within 10 days after cell infusion. This type of life-threatening complication is mainly attributed to uncontrolled release of cytokines from CAR T cells [140]. In addition, exosomes could be delivered through the blood circulation and other body fluids as evidenced by the abundance of exosomes found in most body fluids. Moreover, exosomes have the ability to cross biological barriers such as the blood-brain barrier and blood-tumor barrier as corroborated by the presence of tumor cell-derived exosomes in body fluids [140]. The fact that such a cell-free "exosome therapy" involves the on-bench stimulation and expansion of the effector CAR T cells [26] may allow the genetic modification and/or pharmacologic treatment of the cultured cells in order to increase $\mathrm{T}$ cell activation and/or exosome biogenesis, to enhance EV production. In addition, exosome collection and purification ex vivo involve the elimination of effector CAR T cells, but also bystander or contaminating cells, before exosome infusion [26]. Thus, this strategy would circumvent the undesirable possibility of transducing, for instance, CAR to residual tumoral cells during T cell manufacturing, that may lead to induction of resistance to CAR T cell therapy by unintentional transduction of a single leukemic B cell, as reported [145]. These findings illustrate the need for purging residual contaminating tumor cells from engineered CAR T cells or use alternatives such as cell-free, exosome-based therapies.

Considering that it is not known whether CAR signaling or the involvement of any of the exosomal proapoptotic molecules (perforin, granzymes, FasL, etc) mediate the therapeutic effects observed, these findings require further validation and to formally establish the contribution of these molecules, although they strongly suggest the use of exosomes as biomimetic nanovesicles in antitumor therapy [26]. Recent reviews have dealt with the proapoptotic mechanisms evoked by the proapoptotic molecules present in EV on target cells, please refer to these for further details [146] [136].

It is remarkable that most of the strategies directed to unveil the role of the molecular components involved in exosome biogenesis/degradation and/or release in $\mathrm{T}$ cells (ESCRT, tetraspanins, Rabs, MAL, ISGylation, SNAREs, etc.) are mainly based on either the expression of dominant negative mutants or RNA interference, which led in a vast majority of the approaches to EV secretion inhibition [65] [6]. Thus, although these approaches were indeed useful to establish the necessity of diverse components for exosome secretion, very few of these approaches led to an increase of exosome secretion $[21,25,34]$ [147] [65]. In principle, the positive modulation of exosome secretion ex vivo could be an useful strategy to enhance the effectiveness of exosomes for subsequent in vivo applications. Among all the potential approaches designed to enhance on bench exosome secretion, perhaps the regulation of the lipid pathways and their metabolites (DAG, ceramide) involved in exosome biogenesis appears the most feasible approach, due the existence of quite specific pharmacologic agents suitable for exosome induction ex vivo. Thus, increasing DAG levels using DGK inhibitors (i.e. R59949) [25], or ceramide levels using sphingomyelin synthase inhibitors (i.e. D609) [61], which have been shown to increase exosome secretion, may constitute useful tools for this strategy.

\section{Future developments in the field and concluding remarks}

The application of CAR T cell-derived EV will make this modality of cell free-based therapy more clinically controllable, avoiding CRS syndrome and allowing the tuning of EV production by the CAR T cells. However, EV treatment should be considered as a complementary rather than a substituting technique for CAR T cell-based therapy [81]. Remarkably, recent in vivo results support that RN7SL1-containing CAR T cell-derived EV may be transferred to endogenous immune cells and synergize with CAR T cells to enhance their efficacy against tumor cells even when tumor cells lose CAR antigen expression, by activating the endogenous immune system [144]. In addition, it will be necessary to unveil certain unclear aspects regarding EV bioactive cargo and several mechanistical issues. One relevant issue is to address the exact nature (shedding vesicles and/or 
exosomes) of the EV involved in the observed apoptosis of tumor cells. As summarized in Table 1, in most of the cases this issue has not been addressed. Since the pathways for shedding vesicles and exosome secretion share several regulatory components (i.e. ESCRT, nSMase, ceramide), and some of them act in the opposite direction (nSMase and ceramide have positive roles in exosome secretion in contrast to their negative role in shedding vesicle secretion), it is necessary to clarify the exact nature of the EV responsible for the observed effects in order to optimize ex vivo induction and production of the bioactive EV, and to enrich in the relevant EV type or to eliminate bystander EV. Second, it is necessary to establish the nature of the diverse proapoptotic cargo molecules in the EV responsible for apoptosis [135] [136] and the evoked apoptotic mechanisms in the target tumor cells, or their immunostimulatory role [144] [148], since in the published reports to date using CAR T cell-derived exosomes all these aspects have not been formally clarified (Table 1). With this knowledge in hand, it may be possible to design strategies to modify and/or enhance the EV bioactive cargo [144] [148].

Third, the fact that the same type of cells releases distinct exosome subpopulations [112] with unique compositions may elicit differential effects on recipient cells [85]. Further dissection of exosome heterogeneity, together with the identification of the proapoptotic and bioactive cargo molecules will advance our understanding of exosomal biology in health and disease. To this end, the development of multiparameter, high-throughput, single-vesicle based analytical and preparative methods is needed [86]. Indeed, with this knowledge in hand it will be possible, for instance, to enrich the exosome preparations in the exosome subpopulation carrying the bio-active, relevant molecule. NTA, one of the most commonly used technique for EV analysis, does not allow at this moment the high throughput and the multiparametric, simultaneous acquisition of several fluorochrome emissions. Currently, NTA instruments with two different fluorescent channels have been developed to study two fluorescent probes in the same EV preparation (but not simultaneously in the same vesicle) [88], but lack multiparametric and high throughput capabilities. In principle, flowcytometry (FC) is an ideal technique for EV analysis due to its intrinsic high throughput, multiparametric characterization capability, robust statistical analysis of individualized EV and EV preparative potential, but most conventional FC platforms suffer from detection thresholds above $500 \mathrm{~nm}$ [149]. To circumvent this caveat, dedicated FC has been developed using optimized, laboratory-built configurations of commercially available high-end FC [86]. Furthermore, using an optimally configured and customized commercial flow cytometer, fluorescence-activated vesicle sorting (FAVS) was established as a method to analyse and sort exosomes based solely on the presence of endogenous membrane constituents, including EGFR and the exosomal-enriched marker, CD9 [150]. FAVS represents a major technical advance by allowing analysis and sorting of exosomes based on the expression of candidate cell-surface markers. The analysis and purification of EV via FAVS will expand our understanding of these EV and their subpopulations that are altered in diseases states and may help to uncover the molecular mechanisms underlying their biological function [150]. However, complicated manual hardware adaptations, adjustments and calibration prior to use are needed [86] [151]. This fact precludes the generalized use of dedicated FC to EV sorting and analyses. Thus, alternative, recent implementations have been developed including image FC (iFC) and nano FC. Nano FC allows single vesicle detection down to $7 \mathrm{~nm}$ size, and the incorporation of multiparameter fluorescence detection allows sizing and profiling of individual EV as small as $40 \mathrm{~nm}$ [152]. Certain advanced image techniques such as single-molecule localization microscopy (SMLM) [100] may complement nano FC due to SMLM superb EV detection sensitivity and resolution, although this technique fails to determine EV size and concentration [153]. In addition, high resolution image FC (iFC) [154] facilitates the robust detection and quantification of phenotypically distinct, single EV in heterogeneous samples by using different fluorochrome conjugated antibodies [155] and constitutes a new and encouraging approach for EV analyses. Please refer to excellent reviews summarizing and comparing the different EV analysis methods [153] [156]. 
Fourth, it is remarkable that engineered CAR T cells are cultured during 7-9 days in the presence of interleukin-2 to expand the culture, after the initial stimulation with antiCD3/CD28 to facilitate CAR transduction [26] [142] [81]. Subsequently, the induction of exosome secretion requires re-stimulation of the expanded CAR T cells with antigen-expressing cells or anti-CD3/ CD28. The timing of the second stimulation was based on the necessity to return to the resting activation state of CAR T cells, to minimize AICD [26]. In this context, FasL-carrying, exosome-dependent AICD occurs when activated T lymphocytes undergo a too early re-stimulation [28] [25], which indeed could be a caveat since the number of exosome-producing CAR T cells would decrease and thus exosome production. Considering these facts, an adequate timing for re-stimulation, and the enhancement of $\mathrm{T}$ cell activation at this stage, together with positive modulation of exosome biogenesis will potentially be a synergizing strategy for ex vivo exosome production. For instance, the enhancement of CAR T cell activation achieved by DGK inhibition has been proposed as a promising strategy to increase the antitumor efficacy of CAR T cells due to the enhanced anti-tumor effector functions [157]. Moreover, the fact that cell-free "exosome therapy" involves on-bench stimulation and growth expansion of the effector CAR $\mathrm{T}$ cells [26] may allow the genetic modification and/or pharmacologic treatment of the effector cells in culture, directed to increase T cell activation and/or to enhance EV production. In this context, we propose to transduce genes and/or to add pharmacologic enhancers of $\mathrm{T}$ cell activation during the re-stimulation, as a novel strategy to enhance EV production. Since some T lymphocyte activation enhancers are also positive modulators of exosome biogenesis and secretion in T lymphocytes (i.e. DGK inhibitors such as R59949) [119] [21,25,34], they may constitute a promising strategy for cell-free, CAR T cell-derived exosome-based therapies.

Finally, the recent results showing that immunostimulatory RN7SL1 and antigen peptide released in EV by CAR T cells can be used to enhance an endogenous immune response against tumor opens the possibility that CAR T cells can be used to deliver EV armed with RN7SL1 or RN7SL1 together a peptide antigen of choice [144]. Thus, EV from CAR T cells may transport immunostimulatory RN7SL1 or other immunorelevant molecules, such as immunogenic peptides than can stimulate specific memory $\mathrm{T}$ cells to kill tumors, to the tumor microenvironment. A better understanding of the biology of EV secretion, composition and targeting and uptake by target cells is needed (Table 1), exemplified by the unexplained observation of biased RNA delivery to endogenous immune cells but unbiased delivery of peptide antigens to both tumors and immune cells in the tumor microenvironment [144] [148]. In addition, CAR T cells might be armed during CAR transduction with additional proapoptotic or other bioactive molecules that might be delivered by EV. Most human cancers lack adequate neoantigens, potentially limiting the benefit of CAR T cells that can recruit an endogenous immune response. Thus CAR T cells delivering immunostimulators could be armed with selected peptide antigens, or proapoptotic molecules to potentially overcome major barriers impeding CAR T cell efficacy against solid tumors [144].

Author Contributions: V.C. and M.I. conceived the manuscript and the writing of the manuscript and approved its final content. Conceptualization, V.C. and M.I; writing-original draft preparation, M.I.; writing - review and editing, V.C. and M.I.

Funding: This publication is part of the Grant PID2020-114148RB-I00 funded by MCIN/AEI/ $10.13039 / 501100011033$ and by "ERDF A way of making Europe", by the "European Union".

Acknowledgments: The authors apologize for not including some relevant references due to space limitations. We acknowledge all the past and present members of the lab for their generous contribution. We acknowledge the support of the publication fee by the CSIC Open Access Publication Support Initiative through its Unit of Information Resources for Research (URICI).

Conflicts of Interest: The authors declare no conflict of interest. The funders had no role in the design of the study; in the collection, analyses, or interpretation of data; in the writing of the manuscript, or in the decision to publish the results. 
Abbreviations:

\begin{tabular}{|c|c|}
\hline $\mathrm{Ab}$ & Antibody \\
\hline AICD & Activation-induced cell death \\
\hline AP3 & Adaptor protein 3 \\
\hline $\mathrm{APC}$ & Antigen-presenting cell \\
\hline Apo2L & Apo2 ligand (TRAIL) \\
\hline $\mathrm{ARP} 2 / 3$ & Actin-related proteins $2 / 3$ \\
\hline aSMase & Acid sphingomyelinase \\
\hline BCR & B-cell receptor for antigen \\
\hline CAM & Cell adhesion molecules \\
\hline CAR & Chimeric Antigen Receptors \\
\hline cIS & Central region of the immune synapse \\
\hline cSMAC & Central supramolecular activation cluster \\
\hline Dia1 & Diaphanous-related formin 1 \\
\hline $\mathrm{DC}$ & Dendritic cells \\
\hline dSMAC & Distal supramolecular activation cluster \\
\hline CTL & Cytotoxic T lymphocytes \\
\hline DAG & Diacylglycerol \\
\hline DGK $\alpha$ & Diacylglycerol kinase $\alpha$ \\
\hline dSMAC & Distal supramolecular activation cluster \\
\hline EGFR & Epidermal growth factor receptor \\
\hline EM & Electron microscopy \\
\hline ESCRT & Endosomal sorting complex required for traffic \\
\hline $\mathrm{EV}$ & Extracellular vesicles \\
\hline FC & Flow cytometry \\
\hline FAVS & Fluorescence activated vesicle sorter \\
\hline F-actin & Filamentous actin \\
\hline FasL & Fas ligand \\
\hline FDC & Follicular Dendritic cells \\
\hline FMNL1 & Formin-like 1 \\
\hline HER2 & Human epidermal growth factor receptor 2 \\
\hline $\mathrm{HLH}$ & Haemophagocytic lymphohistiocytosis \\
\hline HRS & Hepatocyte growth factor-regulated tyrosine kinase substrate \\
\hline HS1 & Hematopoietic lineage cell-specific protein 1 \\
\hline ICAM-1 & Intercellular Adhesion Molecule-1 \\
\hline IFN & Interferon \\
\hline $\mathrm{iFC}$ & Image flowcytometry \\
\hline ILV & Intraluminal vesicles \\
\hline IS & Immune synapse \\
\hline LAMP-1 & Lysosomal-associated membrane protein 1 \\
\hline LFA1 & Lymphocyte function-associated antigen 1 \\
\hline LBPA & Lyso-bis-phosphatidic acid \\
\hline LYST & Lysosomal trafficking regulator \\
\hline MHC & Major histocompatibility complex \\
\hline miRNA & MicroRNA \\
\hline MVB & Multivesicular bodies \\
\hline MTOC & Microtubule-organizing center \\
\hline NK & Natural killer cell \\
\hline nSMase2 & Neutral sphingomyelinase 2 \\
\hline NTA & Nanoparticle tracking analysis \\
\hline OVA & Ovalbumin \\
\hline
\end{tabular}




\begin{tabular}{cc}
\hline PA & Phosphatidic acid \\
PBL & Peripheral blood lymphocytes \\
SMLM & Single molecule localization microscopy \\
PHA & Phytohemagglutinin \\
PKC & Protein kinase C \\
PKC $\delta$ & Protein kinase C $\delta$ isoform \\
PLC & Phospholipase C \\
PLD & Phospholipase D \\
pMHC & Peptide/MHC complex \\
pSMAC & Peripheral supramolecular activation cluster \\
RN7SL1 & RNA component of signal recognition particle 7SL1 \\
SEE & Staphylococcus enterotoxin E \\
SMAC & Supramolecular activation cluster \\
SMase & Sphingomyelinase \\
SNAP23 & synaptosomal protein 23 \\
SNARE & N-ethylmaleimide-sensitive fusion attachment protein receptor \\
STX & Syntaxin \\
TCR & T-cell receptor for antigen \\
TAGLN2 & Transgelin-2 \\
Th & T helper \\
vesicle-associated & VAMP \\
membrane protein & \\
\hline &
\end{tabular}

\section{References}

1. van Niel, G.; D'Angelo, G.; Raposo, G., Shedding light on the cell biology of extracellular vesicles. Nat Rev Mol Cell Biol 2018, 19, 213-228.

2. Thery, C.; Ostrowski, M.; Segura, E., Membrane vesicles as conveyors of immune responses. Nature reviews. Immunology 2009, 9, 581-593.

3. Lindenbergh, M.F.S.; Stoorvogel, W., Antigen presentation by extracellular vesicles from professional antigen-presenting cells. Annu Rev Immunol 2018, 36, 435-459.

4. Johnstone, R.M.; Adam, M.; Hammond, J.R.; Orr, L.; Turbide, C., Vesicle formation during reticulocyte maturation. Association of plasma membrane activities with released vesicles (exosomes). The Journal of biological chemistry 1987, 262, 9412-9420.

5. Johnstone, R.M., The jeanne manery-fisher memorial lecture 1991. Maturation of reticulocytes: Formation of exosomes as a mechanism for shedding membrane proteins. Biochem Cell Biol 1992, 70, 179-190.

6. Colombo, M.; Raposo, G.; Théry, C., Biogenesis, secretion, and intercellular interactions of exosomes and other extracellular vesicles. Annual Review of Cell and Developmental Biology 2014, 30, 255-289.

7. Babst, M., Mvb vesicle formation: Escrt-dependent, escrt-independent and everything in between. Curr Opin Cell Biol 2011, 23, $452-457$.

8. Scott, C.C.; Vacca, F.; Gruenberg, J., Endosome maturation, transport and functions. Semin Cell Dev Biol 2014, 31, 2-10.

9. Geuze, H.J., The role of endosomes and lysosomes in mhc class ii functioning. Immunol Today 1998, 19, 282-287.

10. Denzer, K.; Kleijmeer, M.J;; Heijnen, H.F.; Stoorvogel, W.; Geuze, H.J., Exosome: From internal vesicle of the multivesicular body to intercellular signaling device. Journal of cell science 2000, 113 Pt 19, 3365-3374.

11. Pan, B.T.; Teng, K.; Wu, C.; Adam, M.; Johnstone, R.M., Electron microscopic evidence for externalization of the transferrin receptor in vesicular form in sheep reticulocytes. The Journal of cell biology 1985, 101, 942-948.

12. Calvo, V.; Izquierdo, M., Inducible polarized secretion of exosomes in $\mathrm{t}$ and $\mathrm{b}$ lymphocytes. Int J Mol Sci 2020, 21.

13. Mathieu, M.; Martin-Jaular, L.; Lavieu, G.; Thery, C., Specificities of secretion and uptake of exosomes and other extracellular vesicles for cell-to-cell communication. Nature cell biology 2019, 21, 9-17.

14. Trams, E.G.; Lauter, C.J.; Salem, N., Jr.; Heine, U., Exfoliation of membrane ecto-enzymes in the form of micro-vesicles. Biochim Biophys Acta 1981, 645, 63-70.

15. Valadi, H.; Ekstrom, K.; Bossios, A.; Sjostrand, M.; Lee, J.J; Lotvall, J.O., Exosome-mediated transfer of mrnas and micrornas is a novel mechanism of genetic exchange between cells. Nature cell biology 2007, 9, 654-659.

16. Robbins, P.D.; Morelli, A.E., Regulation of immune responses by extracellular vesicles. Nature reviews. Immunology 2014, 14, 195208. 
17. Thery, C.; Witwer, K.W.; Aikawa, E.; Alcaraz, M.J.; Anderson, J.D.; Andriantsitohaina, R.; Antoniou, A.; Arab, T.; Archer, F.; Atkin-Smith, G.K., et al., Minimal information for studies of extracellular vesicles 2018 (misev2018): A position statement of the international society for extracellular vesicles and update of the misev2014 guidelines. J Extracell Vesicles 2018, 7, 1535750.

18. Lotvall, J.; Hill, A.F.; Hochberg, F.; Buzas, E.I.; Di Vizio, D.; Gardiner, C.; Gho, Y.S.; Kurochkin, I.V.; Mathivanan, S.; Quesenberry, P., et al., Minimal experimental requirements for definition of extracellular vesicles and their functions: A position statement from the international society for extracellular vesicles. J Extracell Vesicles 2014, 3, 26913.

19. Witwer, K.W.; Soekmadji, C.; Hill, A.F.; Wauben, M.H.; Buzas, E.I.; Di Vizio, D.; Falcon-Perez, J.M.; Gardiner, C.; Hochberg, F.; Kurochkin, I.V., et al., Updating the misev minimal requirements for extracellular vesicle studies: Building bridges to reproducibility. J Extracell Vesicles 2017, 6, 1396823.

20. Kowal, J.; Arras, G.; Colombo, M.; Jouve, M.; Morath, J.P.; Primdal-Bengtson, B.; Dingli, F.; Loew, D.; Tkach, M.; Thery, C., Proteomic comparison defines novel markers to characterize heterogeneous populations of extracellular vesicle subtypes. Proceedings of the National Academy of Sciences of the United States of America 2016, 113, E968-977.

21. Alonso, R.; Mazzeo, C.; Rodriguez, M.C.; Marsh, M.; Fraile-Ramos, A.; Calvo, V.; Avila-Flores, A.; Merida, I.; Izquierdo, M., Diacylglycerol kinase alpha regulates the formation and polarisation of mature multivesicular bodies involved in the secretion of fas ligand-containing exosomes in t lymphocytes. Cell Death Differ 2011, 18, 1161-1173.

22. Mazzeo, C.; Calvo, V.; Alonso, R.; Merida, I.; Izquierdo, M., Protein kinase d1/2 is involved in the maturation of multivesicular bodies and secretion of exosomes in $\mathrm{t}$ and b lymphocytes. Cell Death Differ 2016, 23, 99-109.

23. Witwer, K.W.; Thery, C., Extracellular vesicles or exosomes? On primacy, precision, and popularity influencing a choice of nomenclature. J Extracell Vesicles 2019, 8, 1648167.

24. Blanchard, N.; Lankar, D.; Faure, F.; Regnault, A.; Dumont, C.; Raposo, G.; Hivroz, C., Tcr activation of human $t$ cells induces the production of exosomes bearing the tcr/cd3/zeta complex. Journal of immunology 2002, 168, 3235-3241.

25. Alonso, R.; Rodriguez, M.C.; Pindado, J.; Merino, E.; Merida, I.; Izquierdo, M., Diacylglycerol kinase alpha regulates the secretion of lethal exosomes bearing fas ligand during activation-induced cell death of $\mathrm{t}$ lymphocytes. The Journal of biological chemistry 2005, 280, 28439-28450.

26. Fu, W.; Lei, C.; Liu, S.; Cui, Y.; Wang, C.; Qian, K.; Li, T.; Shen, Y.; Fan, X.; Lin, F., et al., Car exosomes derived from effector car$\mathrm{t}$ cells have potent antitumour effects and low toxicity. Nature communications 2019, 10, 4355.

27. Gould, S.J.; Raposo, G., As we wait: Coping with an imperfect nomenclature for extracellular vesicles. J Extracell Vesicles 2013, 2.

28. Monleon, I.; Martinez-Lorenzo, M.J.; Monteagudo, L.; Lasierra, P.; Taules, M.; Iturralde, M.; Pineiro, A.; Larrad, L.; Alava, M.A.; Naval, J., et al., Differential secretion of fas ligand- or apo2 ligand/tnf-related apoptosis-inducing ligand-carrying microvesicles during activation-induced death of human t cells. Journal of immunology 2001, 167, 6736-6744.

29. Raiborg, C.; Rusten, T.E.; Stenmark, H., Protein sorting into multivesicular endosomes. Curr Opin Cell Biol 2003, 15, 446-455.

30. Ventimiglia, L.N.; Alonso, M.A., Biogenesis and function of t cell-derived exosomes. Front Cell Dev Biol 2016, 4, 84.

31. Nagle, D.L.; Karim, M.A.; Woolf, E.A.; Holmgren, L.; Bork, P.; Misumi, D.J.; McGrail, S.H.; Dussault, B.J., Jr.; Perou, C.M.; Boissy, R.E., et al., Identification and mutation analysis of the complete gene for chediak-higashi syndrome. Nature genetics 1996, 14, 307-311.

32. Mittelbrunn, M.; Gutierrez-Vazquez, C.; Villarroya-Beltri, C.; Gonzalez, S.; Sanchez-Cabo, F.; Gonzalez, M.A.; Bernad, A.; Sanchez-Madrid, F., Unidirectional transfer of microrna-loaded exosomes from t cells to antigen-presenting cells. Nature communications 2011, 2, 282.

33. Quann, E.J.; Merino, E.; Furuta, T.; Huse, M., Localized diacylglycerol drives the polarization of the microtubule-organizing center in t cells. Nat Immunol 2009, 10, 627-635.

34. Alonso, R.; Mazzeo, C.; Merida, I.; Izquierdo, M., A new role of diacylglycerol kinase alpha on the secretion of lethal exosomes bearing fas ligand during activation-induced cell death of $\mathrm{t}$ lymphocytes. Biochimie 2007, 89, 213-221.

35. Chauveau, A.; Le Floc'h, A.; Bantilan, N.S.; Koretzky, G.A.; Huse, M., Diacylglycerol kinase alpha establishes t cell polarity by shaping diacylglycerol accumulation at the immunological synapse. Sci Signal 2014, 7, ra82.

36. Herz, J.; Pardo, J.; Kashkar, H.; Schramm, M.; Kuzmenkina, E.; Bos, E.; Wiegmann, K.; Wallich, R.; Peters, P.J.; Herzig, S., et al., Acid sphingomyelinase is a key regulator of cytotoxic granule secretion by primary t lymphocytes. Nat Immunol 2009, 10, 761768 .

37. Ventimiglia, L.N.; Fernandez-Martin, L.; Martinez-Alonso, E.; Anton, O.M.; Guerra, M.; Martinez-Menarguez, J.A.; Andres, G.; Alonso, M.A., Cutting edge: Regulation of exosome secretion by the integral mal protein in t cells. Journal of immunology 2015, $195,810-814$.

38. Villarroya-Beltri, C.; Baixauli, F.; Mittelbrunn, M.; Fernández-Delgado, I.; Torralba, D.; Moreno-Gonzalo, O.; Baldanta, S.; Enrich, C.; Guerra, S.; Sánchez-Madrid, F., Isgylation controls exosome secretion by promoting lysosomal degradation of mvb proteins. Nature communications 2016, 7, 13588.

39. Clark, R.H.; Stinchcombe, J.C.; Day, A.; Blott, E.; Booth, S.; Bossi, G.; Hamblin, T.; Davies, E.G.; Griffiths, G.M., Adaptor protein 3-dependent microtubule-mediated movement of lytic granules to the immunological synapse. Nat Immunol 2003, 4, 1111-1120.

40. Stinchcombe, J.C.; Barral, D.C.; Mules, E.H.; Booth, S.; Hume, A.N.; Machesky, L.M.; Seabra, M.C.; Griffiths, G.M., Rab27a is required for regulated secretion in cytotoxic t lymphocytes. The Journal of cell biology 2001, 152, 825-834.

41. Daniele, T.; Hackmann, Y.; Ritter, A.T.; Wenham, M.; Booth, S.; Bossi, G.; Schintler, M.; Auer-Grumbach, M.; Griffiths, G.M., A role for rab7 in the movement of secretory granules in cytotoxic t lymphocytes. Traffic 2011, 12, 902-911. 
42. Combs, J.; Kim, S.J.; Tan, S.; Ligon, L.A.; Holzbaur, E.L.; Kuhn, J.; Poenie, M., Recruitment of dynein to the jurkat immunological synapse. Proceedings of the National Academy of Sciences of the United States of America 2006, 103, 14883-14888.

43. Kurowska, M.; Goudin, N.; Nehme, N.T.; Court, M.; Garin, J.; Fischer, A.; de Saint Basile, G.; Ménasché, G., Terminal transport of lytic granules to the immune synapse is mediated by the kinesin-1/slp3/rab27a complex. Blood 2012, 119, 3879-3889.

44. Ritter, A.T.; Asano, Y.; Stinchcombe, J.C.; Dieckmann, N.M.; Chen, B.C.; Gawden-Bone, C.; van Engelenburg, S.; Legant, W.; Gao, L.; Davidson, M.W., et al., Actin depletion initiates events leading to granule secretion at the immunological synapse. Immunity 2015, 42, 864-876.

45. Herranz, G.; Aguilera, P.; Davila, S.; Sanchez, A.; Stancu, B.; Gomez, J.; Fernandez-Moreno, D.; de Martin, R.; Quintanilla, M.; Fernandez, T., et al., Protein kinase c delta regulates the depletion of actin at the immunological synapse required for polarized exosome secretion by t cells. Frontiers in immunology 2019, 10, 851.

46. Bello-Gamboa, A.; Velasco, M.; Moreno, S.; Herranz, G.; Ilie, R.; Huetos, S.; Dávila, S.; Sánchez, A.; Bernardino De La Serna, J.; Calvo, V., et al., Actin reorganization at the centrosomal area and the immune synapse regulates polarized secretory traffic of multivesicular bodies in $\mathrm{t}$ lymphocytes. J Extracell Vesicles 2020, 9, 1759926.

47. Fernández-Hermira, S.; Sanz-Fernández, I.; Botas, M.; Calvo, V.; Izquierdo, M., Analysis of centrosomal area actin reorganization and centrosome polarization upon lymphocyte activation at the immunological synapse. In Methods in cell biology, Academic Press: 2021.

48. Ma, J.S.; Monu, N.; Shen, D.T.; Mecklenbrauker, I.; Radoja, N.; Haydar, T.F.; Leitges, M.; Frey, A.B.; Vukmanovic, S.; Radoja, S., Protein kinase cdelta regulates antigen receptor-induced lytic granule polarization in mouse cd8+ ctl. Journal of immunology 2007, 178, 7814-7821.

49. Quann, E.J.; Liu, X.; Altan-Bonnet, G.; Huse, M., A cascade of protein kinase c isozymes promotes cytoskeletal polarization in $\mathrm{t}$ cells. Nat Immunol 2011, 12, 647-654.

50. Liu, X.; Kapoor, T.M.; Chen, J.K.; Huse, M., Diacylglycerol promotes centrosome polarization in t cells via reciprocal localization of dynein and myosin ii. Proceedings of the National Academy of Sciences of the United States of America 2013, 110, 11976-11981.

51. Dressel, R.; Elsner, L.; Novota, P.; Kanwar, N.; Fischer von Mollard, G., The exocytosis of lytic granules is impaired in vti1b- or vamp8-deficient ctl leading to a reduced cytotoxic activity following antigen-specific activation. Journal of immunology 2010, 185, 1005-1014.

52. Spessott, W.A.; Sanmillan, M.L.; Kulkarni, V.V.; McCormick, M.E.; Giraudo, C.G., Syntaxin 4 mediates endosome recycling for lytic granule exocytosis in cytotoxic t-lymphocytes. Traffic 2017, 18, 442-452.

53. Pattu, V.; Qu, B.; Marshall, M.; Becherer, U.; Junker, C.; Matti, U.; Schwarz, E.C.; Krause, E.; Hoth, M.; Rettig, J., Syntaxin7 is required for lytic granule release from cytotoxic t lymphocytes. Traffic 2011, 12, 890-901.

54. Bhat, S.S.; Friedmann, K.S.; Knörck, A.; Hoxha, C.; Leidinger, P.; Backes, C.; Meese, E.; Keller, A.; Rettig, J.; Hoth, M., et al., Syntaxin 8 is required for efficient lytic granule trafficking in cytotoxic t lymphocytes. Biochim Biophys Acta 2016, 1863, 16531664.

55. zur Stadt, U.; Schmidt, S.; Kasper, B.; Beutel, K.; Diler, A.S.; Henter, J.I.; Kabisch, H.; Schneppenheim, R.; Nürnberg, P.; Janka, G., et al., Linkage of familial hemophagocytic lymphohistiocytosis (fhl) type-4 to chromosome 6q24 and identification of mutations in syntaxin 11. Human molecular genetics 2005, 14, 827-834.

56. Choudhuri, K.; Llodra, J.; Roth, E.W.; Tsai, J.; Gordo, S.; Wucherpfennig, K.W.; Kam, L.C.; Stokes, D.L.; Dustin, M.L., Polarized release of t-cell-receptor-enriched microvesicles at the immunological synapse. Nature 2014, 507, 118-123.

57. Tchernev, V.T.; Mansfield, T.A.; Giot, L.; Kumar, A.M.; Nandabalan, K.; Li, Y.; Mishra, V.S.; Detter, J.C.; Rothberg, J.M.; Wallace, M.R., et al., The chediak-higashi protein interacts with snare complex and signal transduction proteins. Molecular medicine (Cambridge, Mass.) 2002, 8, 56-64.

58. de Saint Basile, G.; Menasche, G.; Fischer, A., Molecular mechanisms of biogenesis and exocytosis of cytotoxic granules. Nature reviews. Immunology 2010, 10, 568-579.

59. Marsh, M.; van Meer, G., Cell biology. No escrts for exosomes. Science 2008, 319, 1191-1192.

60. Trajkovic, K.; Hsu, C.; Chiantia, S.; Rajendran, L.; Wenzel, D.; Wieland, F.; Schwille, P.; Brugger, B.; Simons, M., Ceramide triggers budding of exosome vesicles into multivesicular endosomes. Science 2008, 319, 1244-1247.

61. Tsutsumi, R.; Hori, Y.; Seki, T.; Kurauchi, Y.; Sato, M.; Oshima, M.; Hisatsune, A.; Katsuki, H., Involvement of exosomes in dopaminergic neurodegeneration by microglial activation in midbrain slice cultures. Biochem Biophys Res Commun 2019, 511, 427-433.

62. Kobayashi, T.; Stang, E.; Fang, K.S.; de Moerloose, P.; Parton, R.G.; Gruenberg, J., A lipid associated with the antiphospholipid syndrome regulates endosome structure and function. Nature 1998, 392, 193-197.

63. Matsuo, H.; Chevallier, J.; Mayran, N.; Le Blanc, I.; Ferguson, C.; Faure, J.; Blanc, N.S.; Matile, S.; Dubochet, J.; Sadoul, R., et al., Role of lbpa and alix in multivesicular liposome formation and endosome organization. Science 2004, 303, 531-534.

64. Ghossoub, R.; Lembo, F.; Rubio, A.; Gaillard, C.B.; Bouchet, J.; Vitale, N.; Slavík, J.; Machala, M.; Zimmermann, P., Synteninalix exosome biogenesis and budding into multivesicular bodies are controlled by arf6 and pld2. Nature communications 2014, 5, 3477.

65. Kowal, J.; Tkach, M.; Théry, C., Biogenesis and secretion of exosomes. Current Opinion in Cell Biology 2014, 29, 116-125.

66. Albacete-Albacete, L.; Navarro-Lérida, I.; López, J.A.; Martín-Padura, I.; Astudillo, A.M.; Ferrarini, A.; Van-Der-Heyden, M.; Balsinde, J.; Orend, G.; Vázquez, J., et al., Ecm deposition is driven by caveolin-1-dependent regulation of exosomal biogenesis and cargo sorting. The Journal of cell biology 2020, 219. 
67. van Niel, G.; Charrin, S.; Simoes, S.; Romao, M.; Rochin, L.; Saftig, P.; Marks, M.S.; Rubinstein, E.; Raposo, G., The tetraspanin cd63 regulates escrt-independent and -dependent endosomal sorting during melanogenesis. Dev Cell 2011, 21, 708-721.

68. Hessvik, N.P.; Llorente, A., Current knowledge on exosome biogenesis and release. Cell Mol Life Sci 2018, 75, 193-208.

69. Obino, D.; Farina, F.; Malbec, O.; Saez, P.J.; Maurin, M.; Gaillard, J.; Dingli, F.; Loew, D.; Gautreau, A.; Yuseff, M.I., et al., Actin nucleation at the centrosome controls lymphocyte polarity. Nature communications 2016, 7, 10969.

70. Calvo, V.; Izquierdo, M., Role of actin cytoskeleton reorganization in polarized secretory traffic at the immunological synapse. Frontiers in Cell and Developmental Biology 2021, 9.

71. Stoorvogel, W.; Kleijmeer, M.J.; Geuze, H.J.; Raposo, G., The biogenesis and functions of exosomes. Traffic 2002, 3, $321-330$.

72. van Niel, G.; Porto-Carreiro, I.; Simoes, S.; Raposo, G., Exosomes: A common pathway for a specialized function. J Biochem 2006, 140, 13-21.

73. Keerthikumar, S.; Chisanga, D.; Ariyaratne, D.; Al Saffar, H.; Anand, S.; Zhao, K.; Samuel, M.; Pathan, M.; Jois, M.; Chilamkurti, N., et al., Exocarta: A web-based compendium of exosomal cargo. Journal of Molecular Biology 2016, 428, 688-692.

74. Li, Q.; Wang, H.; Peng, H.; Huyan, T.; Cacalano, N.A., Exosomes: Versatile nano mediators of immune regulation. Cancers (Basel) 2019, 11.

75. Saunderson, S.C.; Schuberth, P.C.; Dunn, A.C.; Miller, L.; Hock, B.D.; MacKay, P.A.; Koch, N.; Jack, R.W.; McLellan, A.D., Induction of exosome release in primary b cells stimulated via cd40 and the il-4 receptor. Journal of immunology 2008, 180, 81468152.

76. Anel, A.; Gallego-Lleyda, A.; de Miguel, D.; Naval, J.; Martinez-Lostao, L., Role of exosomes in the regulation of $\mathrm{t}$-cell mediated immune responses and in autoimmune disease. Cells 2019,8.

77. Andreola, G.; Rivoltini, L.; Castelli, C.; Huber, V.; Perego, P.; Deho, P.; Squarcina, P.; Accornero, P.; Lozupone, F.; Lugini, L., et al., Induction of lymphocyte apoptosis by tumor cell secretion of fasl-bearing microvesicles. The Journal of experimental medicine 2002, 195, 1303-1316.

78. Peters, P.J.; Borst, J.; Oorschot, V.; Fukuda, M.; Krahenbuhl, O.; Tschopp, J.; Slot, J.W.; Geuze, H.J., Cytotoxic t lymphocyte granules are secretory lysosomes, containing both perforin and granzymes. The Journal of experimental medicine 1991, 173, 10991109.

79. Peters, P.J.; Geuze, H.J.; van der Donk, H.A.; Borst, J., A new model for lethal hit delivery by cytotoxic $t$ lymphocytes. Immunol Today 1990, 11, 28-32.

80. Lugini, L.; Cecchetti, S.; Huber, V.; Luciani, F.; Macchia, G.; Spadaro, F.; Paris, L.; Abalsamo, L.; Colone, M.; Molinari, A., et al., Immune surveillance properties of human nk cell-derived exosomes. Journal of immunology 2012, 189, $2833-2842$.

81. Yang, P.; Cao, X.; Cai, H.; Feng, P.; Chen, X.; Zhu, Y.; Yang, Y.; An, W.; Yang, Y.; Jie, J., The exosomes derived from car-t cell efficiently target mesothelin and reduce triple-negative breast cancer growth. Cellular immunology 2021, 360, 104262.

82. Huotari, J.; Helenius, A., Endosome maturation. EMBO J 2011, 30, 3481-3500.

83. Heijnen, H.F.; Schiel, A.E.; Fijnheer, R.; Geuze, H.J.; Sixma, J.J., Activated platelets release two types of membrane vesicles: Microvesicles by surface shedding and exosomes derived from exocytosis of multivesicular bodies and alpha-granules. Blood 1999, 94, 3791-3799.

84. Thery, C.; Zitvogel, L.; Amigorena, S., Exosomes: Composition, biogenesis and function. Nature reviews. Immunology 2002, 2, 569-579.

85. Willms, E.; Johansson, H.J.; Mager, I.; Lee, Y.; Blomberg, K.E.; Sadik, M.; Alaarg, A.; Smith, C.I.; Lehtio, J.; El Andaloussi, S., et al., Cells release subpopulations of exosomes with distinct molecular and biological properties. Sci Rep 2016, 6, 22519.

86. van der Vlist, E.J.; Nolte-'t Hoen, E.N.; Stoorvogel, W.; Arkesteijn, G.J.; Wauben, M.H., Fluorescent labeling of nano-sized vesicles released by cells and subsequent quantitative and qualitative analysis by high-resolution flow cytometry. Nat Protoc 2012, 7, 1311-1326.

87. Mastoridis, S.; Bertolino, G.M.; Whitehouse, G.; Dazzi, F.; Sanchez-Fueyo, A.; Martinez-Llordella, M., Multiparametric analysis of circulating exosomes and other small extracellular vesicles by advanced imaging flow cytometry. Frontiers in immunology 2018, 9 .

88. Panagopoulou, M.S.; Wark, A.W.; Birch, D.J.S.; Gregory, C.D., Phenotypic analysis of extracellular vesicles: A review on the applications of fluorescence. Journal of Extracellular Vesicles 2020, 9, 1710020.

89. Verweij, F.J.; Balaj, L.; Boulanger, C.M.; Carter, D.R.F.; Compeer, E.B.; D’Angelo, G.; El Andaloussi, S.; Goetz, J.G.; Gross, J.C.; Hyenne, V., et al., The power of imaging to understand extracellular vesicle biology in vivo. Nature methods 2021, 18, 1013-1026.

90. Kobayashi, T.; Beuchat, M.H.; Chevallier, J.; Makino, A.; Mayran, N.; Escola, J.M.; Lebrand, C.; Cosson, P.; Kobayashi, T.; Gruenberg, J., Separation and characterization of late endosomal membrane domains. The Journal of biological chemistry 2002, 277, 32157-32164.

91. Sprong, H.; van der Sluijs, P.; van Meer, G., How proteins move lipids and lipids move proteins. Nat Rev Mol Cell Biol 2001, 2, 504-513.

92. Menck, K.; Sönmezer, C.; Worst, T.S.; Schulz, M.; Dihazi, G.H.; Streit, F.; Erdmann, G.; Kling, S.; Boutros, M.; Binder, C., et al., Neutral sphingomyelinases control extracellular vesicles budding from the plasma membrane. J Extracell Vesicles 2017, 6, 1378056.

93. Verderio, C.; Gabrielli, M.; Giussani, P., Role of sphingolipids in the biogenesis and biological activity of extracellular vesicles. J Lipid Res 2018, 59, 1325-1340.

94. Fooksman, D.R.; Vardhana, S.; Vasiliver-Shamis, G.; Liese, J.; Blair, D.A.; Waite, J.; Sacristan, C.; Victora, G.D.; Zanin-Zhorov, A.; Dustin, M.L., Functional anatomy of $t$ cell activation and synapse formation. Annu Rev Immunol 2010, $28,79-105$. 
95. de la Roche, M.; Asano, Y.; Griffiths, G.M., Origins of the cytolytic synapse. Nature reviews. Immunology 2016, 16, 421-432.

96. Xie, J.; Tato, C.M.; Davis, M.M., How the immune system talks to itself: The varied role of synapses. Immunological reviews 2013, 251, 65-79.

97. Huse, M.; Quann, E.J.; Davis, M.M., Shouts, whispers and the kiss of death: Directional secretion in t cells. Nat Immunol 2008, 9 , 1105-1111.

98. Friedl, P.; den Boer, A.T.; Gunzer, M., Tuning immune responses: Diversity and adaptation of the immunological synapse. Nature reviews. Immunology 2005, 5, 532-545.

99. Huse, M., Microtubule-organizing center polarity and the immunological synapse: Protein kinase c and beyond. Frontiers in immunology 2012, 3, 235.

100. Calvo, V.; Izquierdo, M., Imaging polarized secretory traffic at the immune synapse in living t lymphocytes. Frontiers in immunology 2018, 9, 684 .

101. Peters, P.J.; Geuze, H.J.; Van der Donk, H.A.; Slot, J.W.; Griffith, J.M.; Stam, N.J.; Clevers, H.C.; Borst, J., Molecules relevant for $\mathrm{t}$ cell-target cell interaction are present in cytolytic granules of human $\mathrm{t}$ lymphocytes. Eur J Immunol 1989, 19, 1469-1475.

102. Golstein, P.; Griffiths, G.M., An early history of $\mathrm{t}$ cell-mediated cytotoxicity. Nature Reviews Immunology 2018, 18, 527-535.

103. Bossi, G.; Griffiths, G.M., Degranulation plays an essential part in regulating cell surface expression of fas ligand in $t$ cells and natural killer cells. Nat Med 1999, 5, 90-96.

104. Martinez-Lorenzo, M.J.; Anel, A.; Gamen, S.; Monle n, I.; Lasierra, P.; Larrad, L.; Pineiro, A.; Alava, M.A.; Naval, J., Activated human $t$ cells release bioactive fas ligand and apo2 ligand in microvesicles. Journal of immunology 1999, 163, $1274-1281$.

105. Nagata, S., Apoptosis by death factor. Cell 1997, 88, 355-365.

106. Ashkenazi, A., Targeting death and decoy receptors of the tumour-necrosis factor superfamily. Nature Reviews Cancer 2002, 2, 420-430.

107. Krammer, P.H.; Arnold, R.; Lavrik, I.N., Life and death in peripheral t cells. Nature reviews. Immunology 2007, 7, $532-542$.

108. Nagata, S.; Suda, T., Fas and fas ligand: Lpr and gld mutations. Immunol Today 1995, 16, 39-43.

109. Dustin, M.L., What counts in the immunological synapse? Mol Cell 2014, 54, 255-262.

110. Dustin, M.L.; Choudhuri, K., Signaling and polarized communication across the $\mathrm{t}$ cell immunological synapse. Annu Rev Cell Dev Biol 2016, 32, 303-325.

111. Mittelbrunn, M.; Sanchez-Madrid, F., Intercellular communication: Diverse structures for exchange of genetic information. Nat Rev Mol Cell Biol 2012, 13, 328-335.

112. van der Vlist, E.J.; Arkesteijn, G.J.; van de Lest, C.H.; Stoorvogel, W.; Nolte-'t Hoen, E.N.; Wauben, M.H., Cd4(+) t cell activation promotes the differential release of distinct populations of nanosized vesicles. J Extracell Vesicles 2012, 1.

113. Saliba, D.G.; Cespedes-Donoso, P.F.; Balint, S.; Compeer, E.B.; Korobchevskaya, K.; Valvo, S.; Mayya, V.; Kvalvaag, A.; Peng, Y.; Dong, T., et al., Composition and structure of synaptic ectosomes exporting antigen receptor linked to functional cd40 ligand from helper t cells. Elife 2019, 8.

114. Stinchcombe, J.; Bossi, G.; Griffiths, G.M., Linking albinism and immunity: The secrets of secretory lysosomes. Science 2004, 305, 55-59.

115. Ménasché, G.; Pastural, E.; Feldmann, J.; Certain, S.; Ersoy, F.; Dupuis, S.; Wulffraat, N.; Bianchi, D.; Fischer, A.; Le Deist, F., et al., Mutations in rab27a cause griscelli syndrome associated with haemophagocytic syndrome. Nature genetics 2000, 25, 173-176.

116. Feldmann, J.; Callebaut, I.; Raposo, G.; Certain, S.; Bacq, D.; Dumont, C.; Lambert, N.; Ouachée-Chardin, M.; Chedeville, G.; Tamary, H., et al., Munc13-4 is essential for cytolytic granules fusion and is mutated in a form of familial hemophagocytic lymphohistiocytosis (fhl3). Cell 2003, 115, 461-473.

117. zur Stadt, U.; Rohr, J.; Seifert, W.; Koch, F.; Grieve, S.; Pagel, J.; Strauss, J.; Kasper, B.; Nürnberg, G.; Becker, C., et al., Familial hemophagocytic lymphohistiocytosis type 5 (fhl-5) is caused by mutations in munc18-2 and impaired binding to syntaxin 11 . American journal of human genetics 2009, 85, 482-492.

118. Stepp, S.E.; Dufourcq-Lagelouse, R.; Le Deist, F.; Bhawan, S.; Certain, S.; Mathew, P.A.; Henter, J.I.; Bennett, M.; Fischer, A.; de Saint Basile, G., et al., Perforin gene defects in familial hemophagocytic lymphohistiocytosis. Science 1999, 286, 1957-1959.

119. Sanjuan, M.A.; Jones, D.R.; Izquierdo, M.; Merida, I., Role of diacylglycerol kinase alpha in the attenuation of receptor signaling. The Journal of cell biology 2001, 153, 207-220.

120. Géminard, C.; de Gassart, A.; Blanc, L.; Vidal, M., Degradation of ap2 during reticulocyte maturation enhances binding of hsc70 and alix to a common site on tfr for sorting into exosomes. Traffic 2004, 5, 181-193.

121. Baietti, M.F.; Zhang, Z.; Mortier, E.; Melchior, A.; Degeest, G.; Geeraerts, A.; Ivarsson, Y.; Depoortere, F.; Coomans, C.; Vermeiren, E., et al., Syndecan-syntenin-alix regulates the biogenesis of exosomes. Nature cell biology 2012, 14, 677-685.

122. Colombo, M.; Moita, C.; van Niel, G.; Kowal, J.; Vigneron, J.; Benaroch, P.; Manel, N.; Moita, L.F.; Théry, C.; Raposo, G., Analysis of escrt functions in exosome biogenesis, composition and secretion highlights the heterogeneity of extracellular vesicles. Journal of cell science 2013, 126, 5553-5565.

123. Stinchcombe, J.C.; Majorovits, E.; Bossi, G.; Fuller, S.; Griffiths, G.M., Centrosome polarization delivers secretory granules to the immunological synapse. Nature 2006, 443, 462-465.

124. Carrasco, S.; Merida, I., Diacylglycerol, when simplicity becomes complex. Trends in biochemical sciences 2007, 32, 27-36.

125. Sanchez, E.; Liu, X.; Huse, M., Actin clearance promotes polarized dynein accumulation at the immunological synapse. PloS one 2019, 14, e0210377.

126. Le Floc'h, A.; Huse, M., Molecular mechanisms and functional implications of polarized actin remodeling at the $t$ cell immunological synapse. Cell Mol Life Sci 2015, 72, 537-556. 
127. Billadeau, D.D.; Nolz, J.C.; Gomez, T.S., Regulation of t-cell activation by the cytoskeleton. Nature reviews. Immunology 2007, 7, 131-143.

128. Gomez, T.S.; Kumar, K.; Medeiros, R.B.; Shimizu, Y.; Leibson, P.J.; Billadeau, Daniel D., Formins regulate the actin-related protein 2/3 complex-independent polarization of the centrosome to the immunological synapse. Immunity 2007, 26, 177-190.

129. Gomez, T.S.; McCarney, S.D.; Carrizosa, E.; Labno, C.M.; Comiskey, E.O.; Nolz, J.C.; Zhu, P.; Freedman, B.D.; Clark, M.R.; Rawlings, D.J., et al., Hs1 functions as an essential actin-regulatory adaptor protein at the immune synapse. Immunity 2006, 24, 741-752.

130. Na, B.-R.; Kim, H.-R.; Piragyte, I.; Oh, H.-M.; Kwon, M.-S.; Akber, U.; Lee, H.-S.; Park, D.-S.; Song, W.K.; Park, Z.-Y., et al., Tagln2 regulates $t$ cell activation by stabilizing the actin cytoskeleton at the immunological synapse. The Journal of cell biology 2015, 209, 143-162.

131. Mastrogiovanni, M.; Juzans, M.; Alcover, A.; Di Bartolo, V., Coordinating cytoskeleton and molecular traffic in t cell migration, activation, and effector functions. Frontiers in Cell and Developmental Biology 2020, 8.

132. Douanne, T.; Griffiths, G.M., Cytoskeletal control of the secretory immune synapse. Current Opinion in Cell Biology 2021, 71, 8794.

133. Ueda, H.; Morphew, M.K.; McIntosh, J.R.; Davis, M.M., Cd4+ t-cell synapses involve multiple distinct stages. Proceedings of the National Academy of Sciences of the United States of America 2011, 108, 17099-17104.

134. Chemin, K.; Bohineust, A.; Dogniaux, S.; Tourret, M.; Guegan, S.; Miro, F.; Hivroz, C., Cytokine secretion by cd4+ t cells at the immunological synapse requires cdc42-dependent local actin remodeling but not microtubule organizing center polarity. Journal of immunology 2012, 189, 2159-2168.

135. Wen, C.; Seeger, R.C.; Fabbri, M.; Wang, L.; Wayne, A.S.; Jong, A.Y., Biological roles and potential applications of immune cellderived extracellular vesicles. J Extracell Vesicles 2017, 6, 1400370.

136. Del Vecchio, F.; Martinez-Rodriguez, V.; Schukking, M.; Cocks, A.; Broseghini, E.; Fabbri, M., Professional killers: The role of extracellular vesicles in the reciprocal interactions between natural killer, cd8+ cytotoxic t-cells and tumour cells. J Extracell Vesicles 2021, 10, e12075.

137. Vergani, E.; Daveri, E.; Vallacchi, V.; Bergamaschi, L.; Lalli, L.; Castelli, C.; Rodolfo, M.; Rivoltini, L.; Huber, V., Extracellular vesicles in anti-tumor immunity. Seminars in Cancer Biology 2021.

138. June, C.H.; O'Connor, R.S.; Kawalekar, O.U.; Ghassemi, S.; Milone, M.C., Car t cell immunotherapy for human cancer. Science 2018, 359, 1361-1365.

139. Chan, J.D.; Lai, J.; Slaney, C.Y.; Kallies, A.; Beavis, P.A.; Darcy, P.K., Cellular networks controlling t cell persistence in adoptive cell therapy. Nature Reviews Immunology 2021.

140. Tang, X.J.; Sun, X.Y.; Huang, K.M.; Zhang, L.; Yang, Z.S.; Zou, D.D.; Wang, B.; Warnock, G.L.; Dai, L.J.; Luo, J., Therapeutic potential of car-t cell-derived exosomes: A cell-free modality for targeted cancer therapy. Oncotarget 2015, 6, 44179-44190.

141. Xu, Q.; Zhang, Z.; Zhao, L.; Qin, Y.; Cai, H.; Geng, Z.; Zhu, X.; Zhang, W.; Zhang, Y.; Tan, J., et al., Tropism-facilitated delivery of crispr/cas9 system with chimeric antigen receptor-extracellular vesicles against b-cell malignancies. Journal of controlled release : official journal of the Controlled Release Society 2020, 326, 455-467.

142. Aharon, A.; Horn, G.; Bar-Lev, T.H.; Zagagi Yohay, E.; Waks, T.; Levin, M.; Deshet Unger, N.; Avivi, I.; Globerson Levin, A., Extracellular vesicles derived from chimeric antigen receptor-t cells: A potential therapy for cancer. Human gene therapy 2021, 32, 1224-1241.

143. Haque, S.; Vaiselbuh, S.R., Cd19 chimeric antigen receptor-exosome targets cd19 positive b-lineage acute lymphocytic leukemia and induces cytotoxicity. Cancers (Basel) 2021, 13.

144. Johnson, L.R.; Lee, D.Y.; Eacret, J.S.; Ye, D.; June, C.H.; Minn, A.J., The immunostimulatory rna rn7sl1 enables car-t cells to enhance autonomous and endogenous immune function. Cell 2021, 184, 4981-4995 e4914.

145. Ruella, M.; Xu, J.; Barrett, D.M.; Fraietta, J.A.; Reich, T.J.; Ambrose, D.E.; Klichinsky, M.; Shestova, O.; Patel, P.R.; Kulikovskaya, I., et al., Induction of resistance to chimeric antigen receptor t cell therapy by transduction of a single leukemic b cell. Nat Med 2018, 24, 1499-1503.

146. Wen, C.; Seeger, R.C.; Fabbri, M.; Wang, L.; Wayne, A.S.; Jong, A.Y., Biological roles and potential applications of immune cellderived extracellular vesicles. Journal of extracellular vesicles 2017, 6, 1400370-1400370.

147. Sinha, S.; Hoshino, D.; Hong, N.H.; Kirkbride, K.C.; Grega-Larson, N.E.; Seiki, M.; Tyska, M.J.; Weaver, A.M., Cortactin promotes exosome secretion by controlling branched actin dynamics. The Journal of cell biology 2016, 214, 197-213.

148. Nutt, W.S.; Srivastava, S., Special delivery! Car-t cells transport rn7sl1 to the tumor microenvironment. Trends in Molecular Medicine 2021, 27, 1019-1021.

149. Erdbrügger, U.; Lannigan, J., Analytical challenges of extracellular vesicle detection: A comparison of different techniques. Cytometry. Part A : the journal of the International Society for Analytical Cytology 2016, 89, 123-134.

150. Higginbotham, J.N.; Zhang, Q.; Jeppesen, D.K.; Scott, A.M.; Manning, H.C.; Ochieng, J.; Franklin, J.L.; Coffey, R.J., Identification and characterization of egf receptor in individual exosomes by fluorescence-activated vesicle sorting. J Extracell Vesicles 2016, 5 , 29254.

151. Pospichalova, V.; Svoboda, J.; Dave, Z.; Kotrbova, A.; Kaiser, K.; Klemova, D.; Ilkovics, L.; Hampl, A.; Crha, I.; Jandakova, E., et al., Simplified protocol for flow cytometry analysis of fluorescently labeled exosomes and microvesicles using dedicated flow cytometer. J Extracell Vesicles 2015, 4, 25530.

152. Tian, Y.; Ma, L.; Gong, M.; Su, G.; Zhu, S.; Zhang, W.; Wang, S.; Li, Z.; Chen, C.; Li, L., et al., Protein profiling and sizing of extracellular vesicles from colorectal cancer patients via flow cytometry. ACS nano 2018, 12, 671-680. 
153. Silva, A.M.; Lázaro-Ibáñez, E.; Gunnarsson, A.; Dhande, A.; Daaboul, G.; Peacock, B.; Osteikoetxea, X.; Salmond, N.; Friis, K.P.; Shatnyeva, O., et al., Quantification of protein cargo loading into engineered extracellular vesicles at single-vesicle and singlemolecule resolution. Journal of Extracellular Vesicles 2021, 10, e12130.

154. Erdbrügger, U.; Rudy, C.K.; Etter, M.E.; Dryden, K.A.; Yeager, M.; Klibanov, A.L.; Lannigan, J., Imaging flow cytometry elucidates limitations of microparticle analysis by conventional flow cytometry. Cytometry. Part A : the journal of the International Society for Analytical Cytology 2014, 85, 756-770.

155. Görgens, A.; Bremer, M.; Ferrer-Tur, R.; Murke, F.; Tertel, T.; Horn, P.A.; Thalmann, S.; Welsh, J.A.; Probst, C.; Guerin, C., et al., Optimisation of imaging flow cytometry for the analysis of single extracellular vesicles by using fluorescence-tagged vesicles as biological reference material. J Extracell Vesicles 2019, 8, 1587567.

156. Vogel, R.; Savage, J.; Muzard, J.; Della Camera, G.; Vella, G.; Law, A.; Marchioni, M.; Mehn, D.; Geiss, O.; Peacock, B., et al., Measuring particle concentration of multimodal synthetic reference materials and extracellular vesicles with orthogonal techniques: Who is up to the challenge? Journal of Extracellular Vesicles 2021, 10, e12052.

157. Chan, J.D.; Lai, J.; Slaney, C.Y.; Kallies, A.; Beavis, P.A.; Darcy, P.K., Cellular networks controlling t cell persistence in adoptive cell therapy. Nature reviews. Immunology 2021. 